\title{
छs \\ Superconducting spoke cavities for high-velocity applications
}

\author{
C. S. Hopper* and J. R. Delayen ${ }^{\dagger}$ \\ Center for Accelerator Science, Department of Physics, Old Dominion University, Norfolk, Virginia 23529, USA, \\ and Accelerator Division, Thomas Jefferson National Accelerator Facility, Newport News, Virginia 23606, USA
}

(Received 12 July 2013; published 3 October 2013)

To date, superconducting spoke cavities have been designed, developed, and tested for particle velocities up to $\beta_{0} \sim 0.6$, but there is a growing interest in possible applications of multispoke cavities for high-velocity applications. We have explored the design parameter space for low-frequency, highvelocity, double-spoke superconducting cavities in order to determine how each design parameter affects the electromagnetic properties, in particular the surface electromagnetic fields and the shunt impedance. We present detailed design for cavities operating at 325 and $352 \mathrm{MHz}$ and optimized for $\beta_{0}=0.82$ and 1 .

DOI: 10.1103/PhysRevSTAB.16.102001

PACS numbers: $29.20 .-\mathrm{c}$

\section{INTRODUCTION}

One of the first applications of superconducting radio frequency (SRF) technology to particle accelerators was for a proton accelerator [1] and, until the late 1980s, superconducting accelerating cavities were separated into two distinct velocity classes. The low-velocity structures, designed for the acceleration of protons and heavy ions, extended to the $\beta_{0}=v_{0} / c \sim 0.2$ regime. They were usually based on resonant transmission lines and are often referred to as TEM structures. Among them are the quarter-wave resonator [2], the coaxial half-wave resonator [3,4], and the spoke resonator [3-6]. The high-velocity cavities were used almost exclusively for the acceleration of electrons or positrons and were restricted to $\beta_{0} \sim 1$. These structures were made of a series of coupled cells operating in the $\mathrm{TM}_{010}$ mode and are therefore referred to as TM structures.

Since the late 1980s there has been a growing interest in higher velocity protons and ions [3,5], and TEM structures have been designed for higher and higher velocities [7], while TM structures have been designed for lower and lower velocities, until the two have overlapped in what is referred to as the medium-velocity region corresponding to $\beta_{0} \sim 0.5-0.6[8,9]$.

The majority of superconducting accelerating structures in use or under development, and included in the medium- $\beta_{0}$ regime, fall under these two broad categories. Accelerating charged particles from $\beta_{0} \sim 0.6$ to $\beta_{0}=1$ is typically accomplished using TM cavities. The TM cavities used in the medium-velocity region are essentially similar to the ones in this high-velocity region, but

\footnotetext{
*chopp002@odu.edu; chrsthop@jlab.org

†jdelayen@odu.edu; delayen@jlab.org
}

Published by the American Physical Society under the terms of the Creative Commons Attribution 3.0 License. Further distribution of this work must maintain attribution to the author(s) and the published article's title, journal citation, and DOI. compressed in the longitudinal direction. For low-frequency, low-velocity applications, elliptical cavities are large and potentially mechanically unstable, but for high-velocity applications, they remain dominant in operational accelerators. The reasons for this include the geometrical simplicity (which has led to good design, modeling, and simulation tools), extensive knowledge base (both in research institutions and industry), and low surface fields at high- $\beta_{0}[10]$. While basic TEM-type cavities, i.e., those with cylindrical loading elements, may also be easy to design, model, and fabricate, the improved mechanical properties (e.g. low microphonics and minimal sensitivity to He pressure) are only accomplished with more advanced engineering and fabrication techniques [11,12].

Spoke and other low-frequency, low-velocity cavities were primarily intended for $4.2 \mathrm{~K}$ operation; however, recent developments in fabrication and processing techniques $[7,13,14]$ have produced results which indicate that 2 K operation may be more economical for large machines, even taking into account the differences in refrigerator efficiency [9]. For smaller machines, on the other hand, $2 \mathrm{~K}$ operation may not be practical, in which case, spoke cavities offer a viable $4.2 \mathrm{~K}$ option [15].

As the interest in TEM structures has increased and their design advanced, more and more spoke cavities have been built and tested [14,16-22]. With the advantages that spoke cavities can offer (which will be discussed later), there is recent interest in the design and development of these structures for the high-velocity regime. Some applications include, but are not limited to, small, low-energy electron accelerators for compact light sources [23,24], nondestructive assay system of nuclear materials in spent fuel using nuclear resonance fluorescence [25,26], and GeV-scale proton accelerators such as spallation sources [27]. Spoke cavities have currently been built and tested for up to $\sim \beta_{0}=0.6$ [16], but are now under development for up to $\beta_{0}=1$ [28]. The concept for a $\beta_{0}=1$ double-spoke cavity is shown in Fig. 1. 


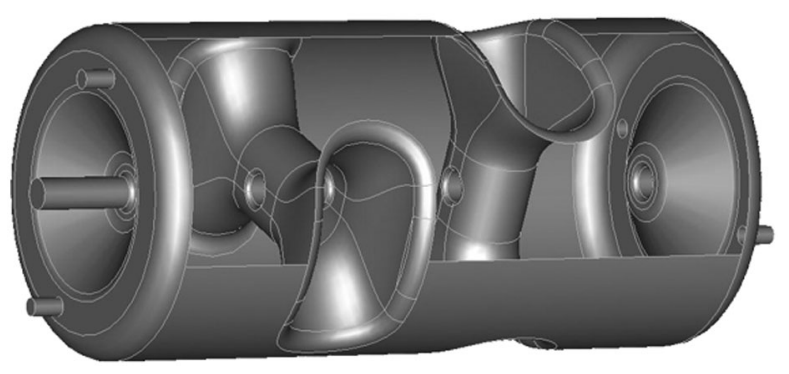

FIG. 1. $325 \mathrm{MHz}, \beta_{0}=1$ double-spoke cavity.

\section{FIGURES OF MERIT}

The performance of superconducting cavities, and what applications they are appropriate for, can often be characterized by a few important properties or parameters. This section gives a brief review of the cavity properties and parameters which will be reported on in the following sections.

The motion of a particle subjected to the electromagnetic fields within a resonant cavity is governed by the Lorentz force,

$$
\vec{F}=\frac{d(m \vec{v})}{d t}=q(\vec{E}+\vec{v} \times \vec{B}),
$$

where $m$ is the relativistic mass of the particle with velocity $\vec{v}$ and charge $q$. Because of the symmetry of the spoke cavity, there is no magnetic field on axis, in the fundamental mode, thus acceleration takes place through the interaction with an electric field parallel to the velocity of the particle.

The energy (or voltage) gain acquired by the particle is the work done by the longitudinal electric field which, under the assumption that the velocity of the particle does not change in the cavity, is given by

$$
V_{\mathrm{acc}}(\beta, \phi)=\int_{-\infty}^{\infty} E_{z} e^{i[(\omega z / \beta c)+\phi]} d z,
$$

where $\omega$ is the rf frequency, $\phi$ is the phase between the particle and the rf field, and $z$ is the direction of particle propagation.

Under the assumption that the velocity does not change within the cavity, the voltage gain $V_{\text {acc }}(\beta, \phi)$ has a sinusoidal dependence on the phase $\phi$ and will have a maximum $V_{0}$ for a certain particle velocity $\beta_{0}$ which will also be used to define the $\beta$ of the cavity. $V_{0}$ can be divided by a reference length $L$ to define the accelerating field at which the cavity is operating,

$$
E_{\text {acc }}=\frac{V_{0}}{L} .
$$

While there is a consensus in the definition of the reference length $L$ for TM cavities, there is no universally agreed upon definition for TEM cavities. In low-velocity TEM cavities, where the gaps are much less than the rf wavelength, the problem is treated as an electrostatic one, and the reference length can be defined as

$$
L=N \frac{\beta_{0} \lambda}{2},
$$

where $N$ is the number of loading elements, $\beta_{0}$ is the velocity of the particle that would acquire the maximum voltage from the cavity, and $\lambda$ is the free-space wavelength corresponding to the frequency of the accelerating mode [29].

In TEM structures, with uniform loading elements, such as a spoke cavity with cylindrical spokes, the voltage acquired by the particle in the end gaps is about half the voltage gained in the central gaps, therefore, it is logical to use $N$ as the number of loading elements. In an optimized cavity, as will be shown later, this is not the case. When the spoke becomes large and noncylindrical, as is the case with the cavities presented here, the voltage gain is almost the same in the end gaps and the central gaps; therefore we find that in the present case, using $N$ as the number of accelerating gaps rather than loading elements allows for a more accurate normalized field comparison between single-spoke and multispoke cavities.

The energy stored in a cavity is given by

$$
U=\frac{1}{2} \epsilon_{0} \int_{V}|\vec{E}|^{2} d V=\frac{1}{2} \mu_{0} \int_{V}|\vec{H}|^{2} d V
$$

where the integration is taken over the entire volume of the cavity. The power dissipation is given by

$$
P_{d}=\frac{1}{2} \int R_{s}|\vec{H}|^{2} d S,
$$

where $R_{s}$ is the surface resistance and the integral is taken over the inner surface of the cavity. The ratio of stored energy and the energy dissipated during one radian in the walls of the cavity is the quality factor,

$$
Q_{0}=\frac{\omega_{0} U}{P_{d}} .
$$

The geometrical factor, which is the product of the quality factor and the surface resistance, $G=R_{s} Q_{0}$ (in $\Omega$ ), is independent of cavity size and material and depends only on the shape of the structure and the electromagnetic mode.

A measure of the power dissipation induced by the $\mathrm{rf}$ currents, and thus the efficiency of the structure in converting rf power to voltage gain, is the shunt impedance defined here as

$$
R=\frac{V_{0}^{2}}{P_{d}}
$$

Since the power dissipation given in Eq. (6) is clearly dependent on the material, for a superconducting structure it has a direct impact on the requirements of the cryogenic system. A material-independent parameter which is often 
quoted and is more useful in assessing a particular cavity design is the product of the shunt impedance and the surface resistance, $R R_{s}$ (in $\Omega^{2}$ ).

The ratio of shunt impedance and quality factor $[R / Q]$, is another parameter that depends only on shape and not on cavity size or material. The parameter $R R_{s}$ is obtained as the product of $[R / Q]$ and $G=Q R_{s}$, and is often used (as we do here) in place of shunt impedance because it does not depend on material properties and operating frequency.

\section{FEATURES OF THE SPOKE CAVITY}

A single-spoke cavity is a variation of the coaxial half-wave geometry; in its fundamental mode of operation, the spoke sustains a TEM mode where the length of the spoke is approximately half of the rf wavelength. The loading elements of multispoke cavities operate out of phase with their nearest neighbor by $\pi$ and, for improved mode separation and lower surface fields, are usually oriented perpendicularly to each other.

We summarize here the features of spoke cavities which make them particularly attractive in the low-velocity to midvelocity regime. These features, as will be shown in the rest of the paper, are still present in the high-velocity regime, although in some cases the advantages TEM cavities offer in other velocity regimes are reduced.

\section{A. Size}

The diameter of a simple spoke cavity (with uniform spokes) is on the order of half the rf wavelength, whereas the diameter of a TM cavity is about twice that. This allows for either smaller physical dimensions at the same operating frequency or close to half the operating frequency for the same physical diameter. While this is true at low $\beta_{0}$ and for simple spoke geometries, higher- $\beta_{0}$-optimized geometries result in this factor of 2 being reduced. Nonetheless, since the BCS surface resistance is proportional to the square of the rf frequency, accelerators can be designed to operate at lower frequencies (and have fewer elements with different fundamental frequencies) where $4.2 \mathrm{~K}$ operation is practical while maintaining cavities of a reasonable size. Furthermore, at half the frequency of a TM cavity of the same $\beta_{0}$, a multispoke cavity of the same length would have half the number of cells. This results in a larger velocity acceptance causing the cavity to be useful over a wider range of velocities. Lower frequency would also lead to a higher longitudinal acceptance, which could prove beneficial in high-current applications.

\section{B. Cell-to-cell coupling}

In elliptical cavities, the cell-to-cell coupling occurs through the iris opening, but in spoke cavities, which are more open, the magnetic field lines couple all cells [30].

The cell-to-cell coupling in multispoke cavities, with simple geometries, is high and they are much more robust as compared to TM cavities with respect to manufacturing inaccuracies. Tuning to achieve field profile balance is important in TM cavities but is usually not necessary in multispoke cavities.

Even in velocity-of-light multispoke cavities, the fundamental mode is the lowest frequency mode, which allows for simpler damping and extraction of higher-order modes. Also, unlike TM cavities, the stronger cell-to-cell coupling together with the small number of spokes, implies that the accelerating mode will be well separated from the nearest mode.

\section{Surface fields and energy content}

As was mentioned previously, the spokes in a multispoke cavity operate out of phase by $\pi$ from each other in a TEM-like mode. As such, the fields are concentrated around the spoke and decay rapidly moving away from them. TM elliptical cavities have a larger volume which is uniformly filled with electromagnetic energy. As a result, spoke cavities tend to have a smaller energy content and higher shunt impedance. The fields on the outer surface can be relatively small; this also allows for both the fundamental power coupler and higher-order mode extraction couplers to be located on the outer surface rather than on the beam line [31-34], which is customary for elliptical cavities.

One disadvantage is that for a given gradient defined by using the inside length of the cavity as the reference length, the peak surface fields are higher in a spoke cavity compared to those in an elliptical cavity, at least in the high- $\beta$ regime [8]. However, in light of the fact that there is no need to use the beam line for couplers in a spoke cavity, it is not clear that the surface fields would be significantly higher at a constant real estate gradient. Additionally, spoke cavities are intended to be used mostly in relatively high-current and/or continuous wave applications where the operating gradients would be modest and not limited by peak surface fields [15].

\section{ELECTROMAGNETIC DESIGN OF HIGH-VELOCITY SPOKE CAVITIES}

High surface fields in superconducting cavities are highly undesirable because of the detrimental effects on performance. At high surface magnetic fields, quenching can occur, and high surface electric fields can cause field emission. When comparing the performance of cavities, normalized surface fields are often discussed. These fields are $E_{p} / E_{\text {acc }}$ and $B_{p} / E_{\text {acc }}$, where $E_{p}$ is the peak surface electric field, $B_{p}$ is the peak surface magnetic field, and $E_{\text {acc }}$ is the accelerating electric field defined in Eq. (3). Minimizing these fields is one of the goals in cavity design. Note that, for all the cavities that we have investigated, the inside length of the cavity (iris-to-iris) is always substantially less than that given by Eq. (4) when $N$ is the number of gaps. Therefore, if we had chosen the inside length as 
the reference length to define $E_{\text {acc }}$, the quoted ratios of peak fields to accelerating field would have been lower. The disadvantage of that choice is that the inside length, even at constant frequency and $\beta_{0}$, is not constant but depends on the details of the cavity geometry, and therefore makes it difficult to compare different designs. Our choice for the reference length is independent of the particularities of a design, once the frequency, $\beta_{0}$, and number of spokes are chosen, and makes comparison easier.

It is important to note that there is no such thing as an "optimal" design for all applications. Each application will have specific design considerations; what may constitute an optimized design for one project may in fact be undesirable for another. An optimized design, however, is more than just minimization of normalized fields since, at some point in the design, both peak surface electric field and peak surface magnetic field cannot be reduced anymore and the reduction of one often leads to the increase of the other. It is then important for the normalized surface fields to be balanced in the sense that the current technical limits or application-specific demands are achieved simultaneously. In other words, if a peak surface electric field of $40 \mathrm{MV} / \mathrm{m}$ and a peak surface magnetic field of $80 \mathrm{mT}$ can be routinely reached with the same probability, then a cavity with a ratio of $B_{p} / E_{p} \simeq 2 \mathrm{mT} /(\mathrm{MV} / \mathrm{m})$ would indicate that the normalized fields are properly balanced. Obviously, this "optimized" ratio is very much dependent on the state of the art, and has changed over time. It is also dependent on the application and the intended operational conditions, such as temperature. For this work we have used as a design goal a ratio of $\simeq 2 \mathrm{mT} /(\mathrm{MV} / \mathrm{m})$.

In some applications, maximization of the shunt impedance is more important than minimization of the surface fields and the designs that maximize the former can be different from the ones that minimize the latter. In other words, there is not a single best optimized design, and what we have attempted here is to show how various design choices (dimensions, shapes) can affect the electromagnetic properties (fields, shunt impedance).

This work has been done using the three-dimensional electromagnetic design software CST MICROWAVE STUDIOC [35]. The design environment allows for a multitude of structures to be simulated and a variety of calculations to be performed. The electromagnetic simulations are accomplished using the finite integration technique [35]. From these simulations, the frequencies and field properties of a desired number of cavity modes can be determined. In addition, the cavity properties described in the previous section can be evaluated.

\section{A. Optimization strategies}

The basic geometry of a spoke cavity consists of an outer conductor, usually either cylindrical or rectangular, with one or more "spokes," which are conductors that run radially, through the longitudinal symmetry axis.
A number of strategies can be used for the optimization process; here we will provide a detailed description of those presented in [28]. Alternatively, advanced methods such as combining a simulation code with a multiobjective genetic algorithm have been used [26]. Multiobjective optimization can rapidly lead to an optimized design but makes it more difficult to separate the impact of each parameter on the cavity properties. Here we have chosen a more systematic approach which results in consistent optimization outcomes to those obtained with a multiobjective approach.

Any optimization strategy will quickly reveal that the geometry of the spokes themselves often has the greatest impact on the peak surface fields. Spokes can be broken into two areas in terms of both their physical location and field (electric or magnetic) that they primarily affect. The region which intersects the wall of the outer conductor is referred to as the spoke base, while the region intersecting the beam line is referred to as the spoke aperture. The base or aperture regions are often referred to as having elliptical, racetrack, or cylindrical geometries. Figure 2 shows the main parameters of spokes with a racetrack geometry. It should be noted that the same definitions apply to elliptical spoke geometries.

Performing a sweep of the parameters identified in Fig. 2, whether the spoke aperture and base are of the elliptical or racetrack variety, can be done by decoupling the size from the shape. By doing so, desirable shapes can be found and the best sizes of those can be determined. Alternatively, both the size and shape can be varied simultaneously.

In order to identify a shape which effectively minimizes the surface fields, several different values for spoke base (aperture) area or circumference can be chosen and a sweep of two parameters can be done while maintaining that area or circumference. For example, the base width and length can be varied while maintaining a constant circumference. This has the effect of changing the base

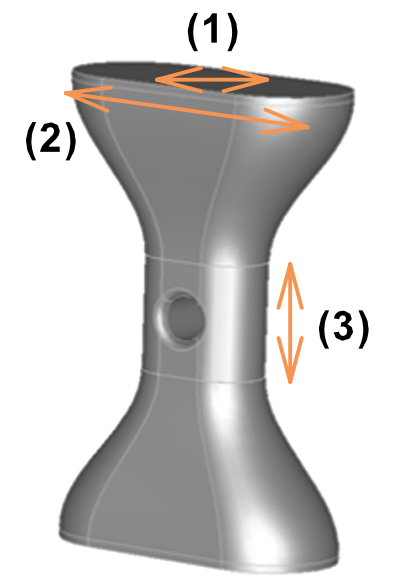

FIG. 2. CST MICROWAVE STUDIOC (MWS) view of a racetrackshaped spoke. (1) base or aperture width, (2) base or aperture length, (3) aperture height. 
shape while keeping the overall surface area in contact with the fields unchanged. Doing so allows us to distinguish shapes that are more desirable than others. With a variety of preferred shapes identified, the optimal sizes of these shapes can be obtained and compared by scaling the dimensions while maintaining the shape.

In addition to the geometries explored, it is important to investigate the orientation of the spokes relative to the beam line. We define a spoke base or aperture region as being longitudinal if the longest dimension (either racetrack or elliptical geometries) is parallel to the beam line and transverse otherwise. More will be said about this in the next section. The data presented here, unless otherwise stated, was obtained while performing an optimization of a two-spoke, $325 \mathrm{MHz}, \beta_{0}=1$ cavity.

\section{B. Dependence of $\mathrm{rf}$ properties on geometric parameters}

In this section, we analyze dependencies that the peak surface fields and shunt impedance have on various cavity dimensions. It should be understood that what is described here are general trends; the actual magnitudes of variations will depend greatly on the values of the cavity parameters which remain fixed.

The cavity radius and iris-to-iris length are approximately determined by the operating frequency and desired $\beta_{0}$. The peak surface fields depend greatly on the shape and dimensions of both the spoke base and the spoke aperture region. All data presented have been acquired under conditions of constant frequency and $\beta_{0}$, meaning that the cavity radius and iris-to-iris length were varied along with the parameters under consideration in order to maintain constant frequency and design velocity.

As was already mentioned, a reference length given by Eq. (4), where $N$ is the number of full accelerating gaps, is used here. For high-velocity spoke cavities, we found that if a single-spoke cavity is assumed to have two accelerating gaps and a two-spoke cavity has three full accelerating gaps, then, for identical spoke and end-cap geometries, normalized surface fields and shunt impedance are consistent and can be accurately compared. Figure 3 shows an example of the dependence of the normalized magnetic field on the spoke base length. The cavities are the same in all respects except iris-to-iris length and outer conductor diameter.

Figure 4 shows some additional cavity parameters discussed here. Both the spoke base and aperture region have been investigated with elliptical, cylindrical, and racetrack geometries. The parameters in Figs. 2 and 4 will be discussed in terms of how they influence the normalized fields, shunt impedance, energy content, and frequency. There are no significant differences in terms of peak surface fields and shunt impedance achievable with elliptical or racetrack geometries, so here we have chosen to present the optimization results for a racetrack spoke base geometry.

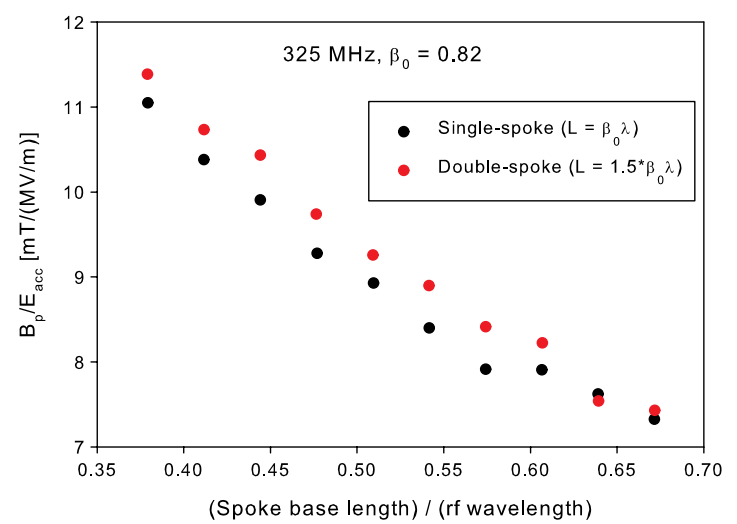

FIG. 3. Dependence of the normalized magnetic field on the spoke base length for a single- and double-spoke cavity with the same spoke and end-cap geometries.

\section{Spoke base}

The magnetic field of the fundamental accelerating mode in a spoke cavity is more concentrated near the surface of the outer conductor and encircles the spokes; the size and shape of the spoke base region thus has a strong effect on the peak surface magnetic field and a lesser effect of the peak surface electric field.

A comparison of the normalized magnetic fields that longitudinal and transverse spoke base orientations provide is presented in Fig. 5(a). The dependence of the normalized field on the longest base dimension (which we call the length) is similar, at smaller dimensions, for both orientations; however, a lower normalized field can be obtained with a larger transverse base. The difference, for this given cavity's other fixed dimensions, is about $25 \%$, which is significant. An even greater difference can be seen in Fig. 5(b). A transverse spoke base can provide a shunt impedance which far exceeds that of a longitudinal base. At a value of spoke base length to rf wavelength of 0.6 , for example, a transverse spoke base can provide a shunt impedance on the order of $70 \%$ higher than a longitudinally oriented spoke base. In a small machine, intended to operate at $4 \mathrm{~K}$, this difference in shunt impedance would have a dramatic impact on the power dissipation and thus the cooling requirements.

Additionally, the energy content in a longitudinal orientation can be close to $50 \%$ higher than its transverse

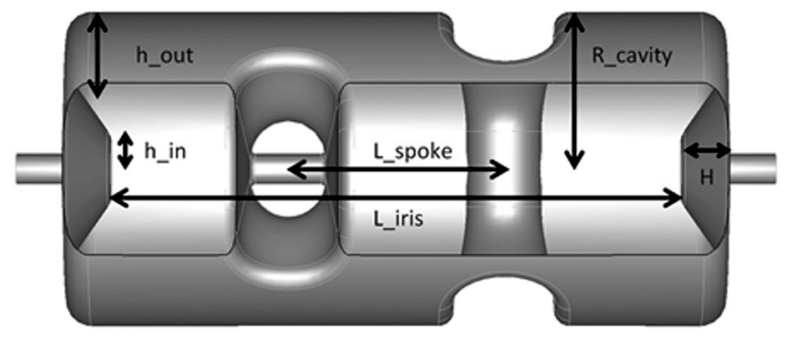

FIG. 4. Cut-away view of a two-spoke $325 \mathrm{MHz}, \beta_{0}=1$ cavity. 

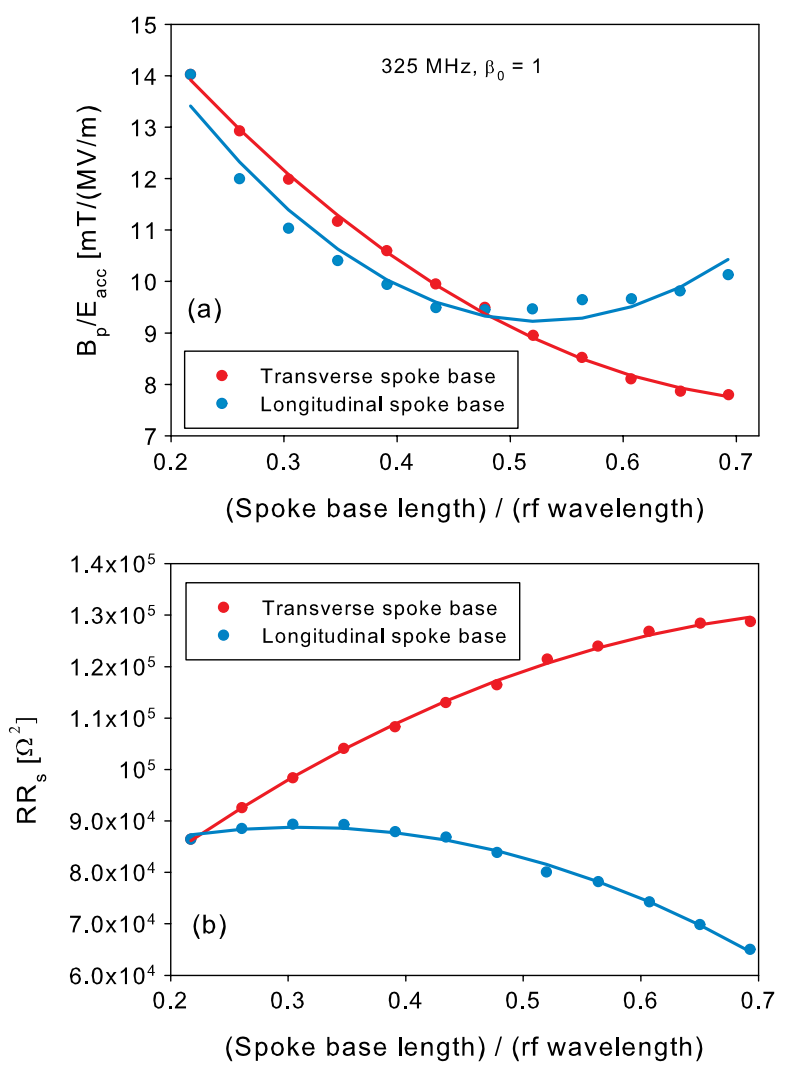

FIG. 5. (a) Dependence of $B_{p} / E_{\text {acc }}$ and (b) $R R_{s}$ on spoke base length normalized to $\mathrm{rf}$ wavelength for a $325 \mathrm{MHz}, \beta_{0}=1$ double-spoke cavity at a constant base width of $200 \mathrm{~mm}$. The blue curves represents a longitudinal base geometry, while the red curves are for an identical spoke base, but oriented transversely to the beam line. In both plots, the leftmost point is common to both curves since it represents a cylindrical spoke base.

counterpart. This is illustrated in Fig. 6. In terms of microphonics control, the higher energy content of a longitudinally oriented spoke base could be much more problematic [36].

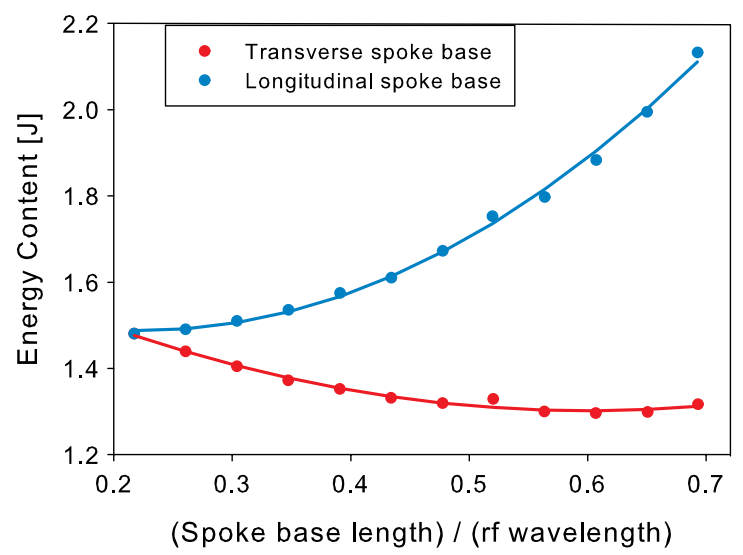

FIG. 6. Dependence of energy content on spoke base length for longitudinal and transverse orientations at a constant accelerating field of $1 \mathrm{MV} / \mathrm{m}$. The leftmost point is common to both curves since it represents a cylindrical spoke base.

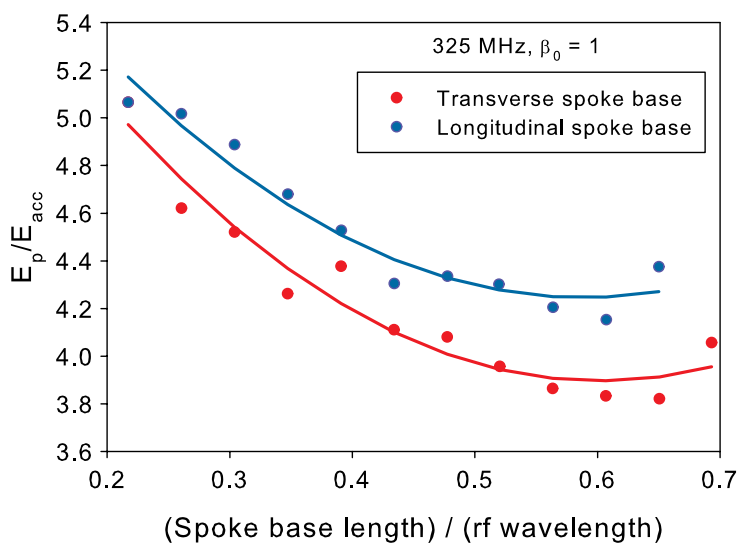

FIG. 7. Dependence of normalized electric field on spoke base length for longitudinal and transverse orientations. The leftmost point is common to both curves since it represents a cylindrical spoke base.

For the two orientations, the difference in the achievable normalized electric field is not as much as for the other $\mathrm{rf}$ properties. Figure 7 shows this difference to be at most about $10 \%$. It must be stressed, however, that these results by no means suggest that a longitudinal orientation should not be considered. As will be shown later, the longitudinal base results in a smaller cavity diameter for a given frequency and a stronger cell-to-cell coupling (i.e. increased mode separation). For applications where shunt impedance is not critical, but size and large mode separation are, the longitudinal orientation may be preferable.

Before proceeding with any optimization strategy, it is important to first understand how each of the individual parameters affect the cavity's rf properties. Figures 5-7 have already shown the dependence that several cavity properties have on the spoke base length. Figure 8 shows a parameter sweep of the spoke base width (transverse and longitudinal racetrack geometry) to show how the normalized magnetic and electric fields are affected, while Fig. 9 shows how the shunt impedance and energy content change. Note that in Figs. 8 and 9, the spoke base approaches a cylinder, which would be located far to the right on the plots, but never actually becomes cylindrical. This is because at this base length, a cylindrical base would be impractical and thus not relevant.

Figure 8 illustrates that the base width orientation can only improve the normalized electric field by about $10 \%$, while the impact on the magnetic field is higher. Figure 9(a) shows that increasing the spoke base width can have a positive effect on the shunt impedance for both orientations, but more so for the longitudinal base. This is consistent with Fig. 5, which showed that a longitudinal base has a higher shunt impedance for a more cylindrical shape. In Fig. 9(b), it can be seen that the energy content decreases for a wider longitudinal base, and increases for the transverse orientation. Again, this is consistent with what was illustrated in Fig. 5 which showed that a more cylindrical shape provides a lower energy content than a longitudinal base. 

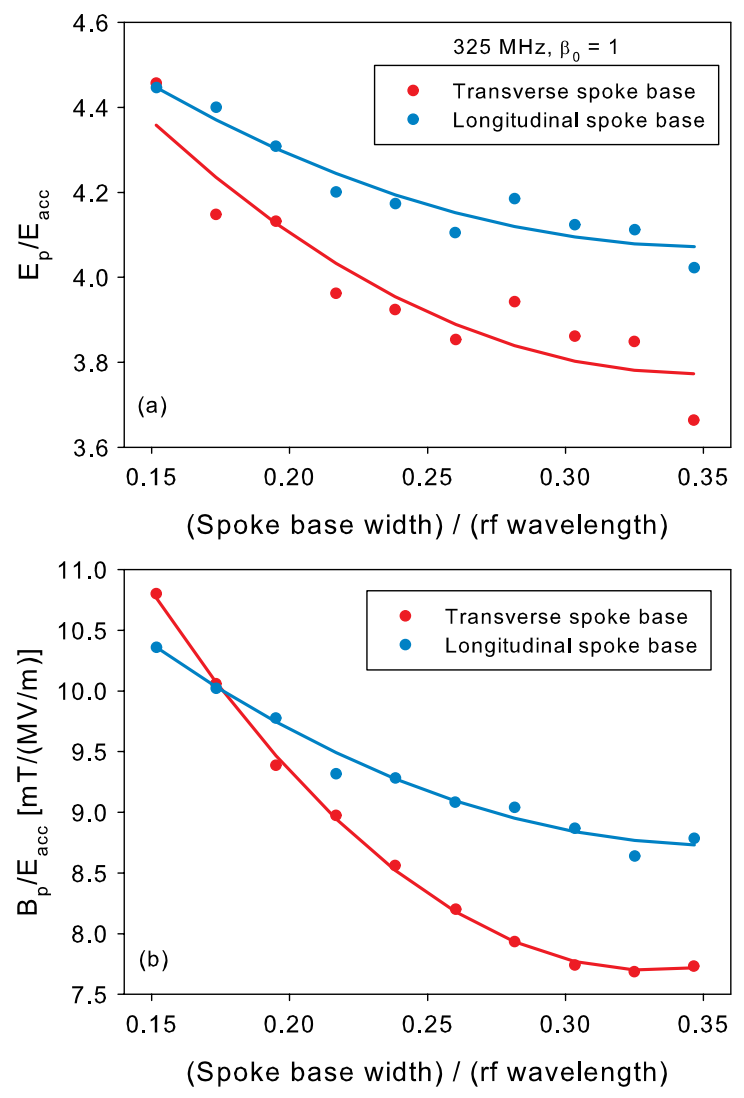

FIG. 8. (a) Dependence of $E_{p} / E_{\text {acc }}$ and (b) $B_{p} / E_{\text {acc }}$ on the spoke base width at a constant base length of $480 \mathrm{~mm}$.

As mentioned in the previous section, shapes can be chosen and the sizes can be varied until an optimum geometry is found. In order to eliminate certain shapes for consideration, balancing of the fields should be considered. Figure 10 is a plot of $B_{p}$ vs $E_{p}$ for several spoke base shapes. It is clear that many of these shapes need not be investigated further. For designs that fall close to the lines representing balanced fields, the optimal sizes are found and compared.

When further optimizing cavities which exhibit wellbalanced fields, it must be emphasized that this strategy is intended for optimization of a given shape, and therefore represent local maxima and minima in shunt impedance and normalized fields, respectively. This is the reason that the effects that individual cavity dimensions have on the optimization parameters should be understood. Finding the dimensions which produce these local maxima and minima can then serve as a starting point for further modification of the shape to either enhance the shunt impedance or decrease the surface fields.

Taking some of the cavities which exhibit balanced fields from Fig. 10, and keeping the shape relatively constant (by fixing the ratio of base width to length, for example), an optimized size can be found. Figures 12 and 13 show the dependence of normalized fields, shunt impedance, and energy content on spoke base size for three different shapes. These shapes are depicted in Fig. 11.
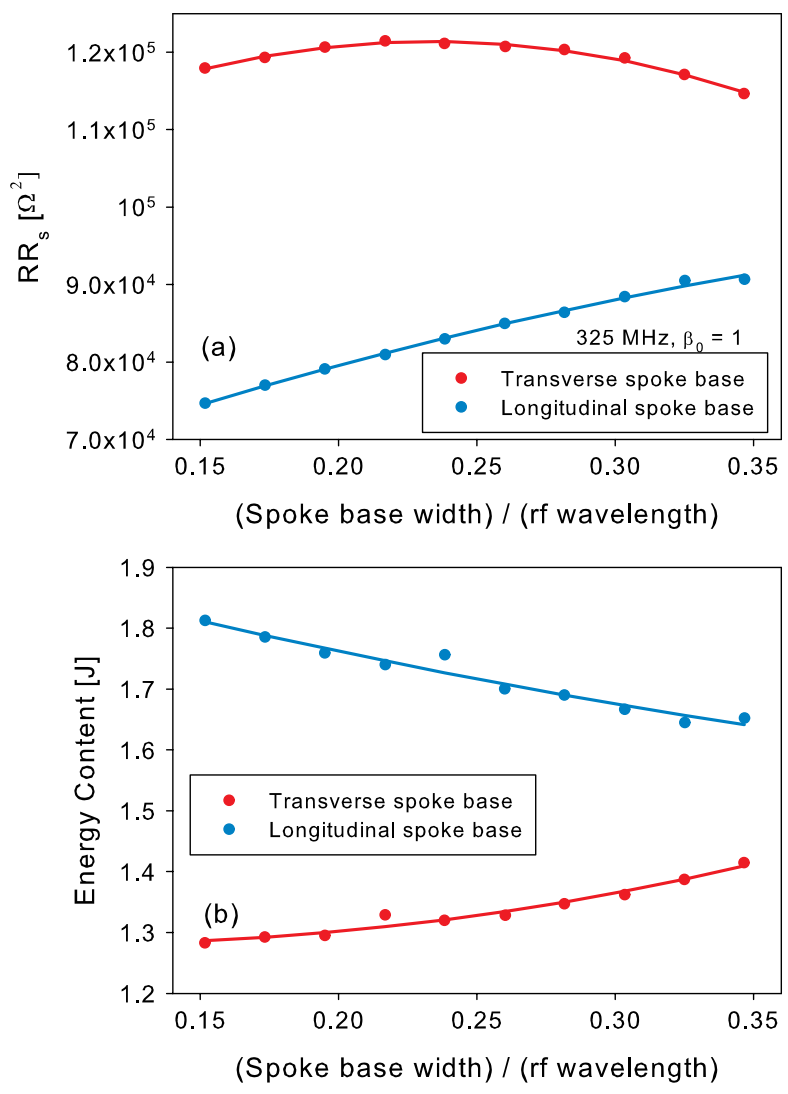

FIG. 9. (a) Dependence of $R R_{s}$ and (b) energy content (at $1 \mathrm{MV} / \mathrm{m}$ ) on the spoke base width.

It is clear from Fig. 12 that, in general, a larger spoke base decreases the normalized fields. The difference in achievable fields, for a given shape, is less for a base where the width and length dimensions are closer [shape (c) in Fig. 11]. From Fig. 13, a larger base generally leads to a higher shunt impedance. For larger width/length ratios, the differences in achievable shunt impedance and energy content are quite significant. If a high shunt impedance is

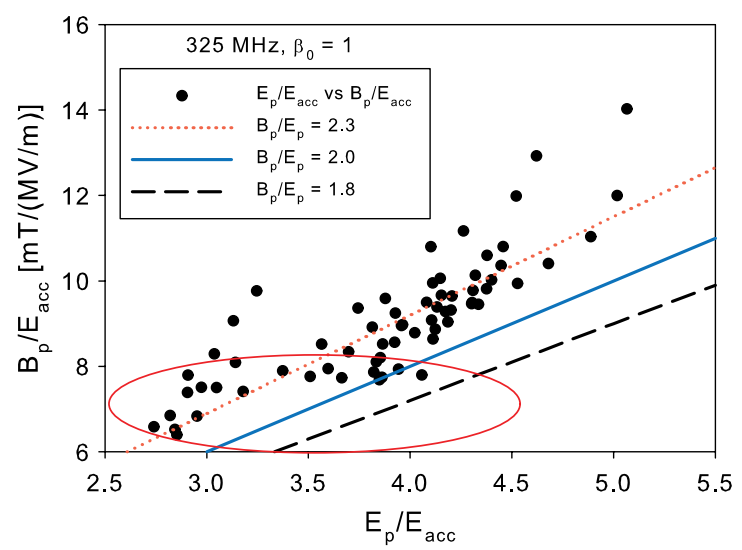

FIG. 10. $B_{p} / E_{\text {acc }}$ vs $E_{p} / E_{\text {acc }}$ for various spoke base shapes. Those shapes within the circled region are candidates for further investigation. 


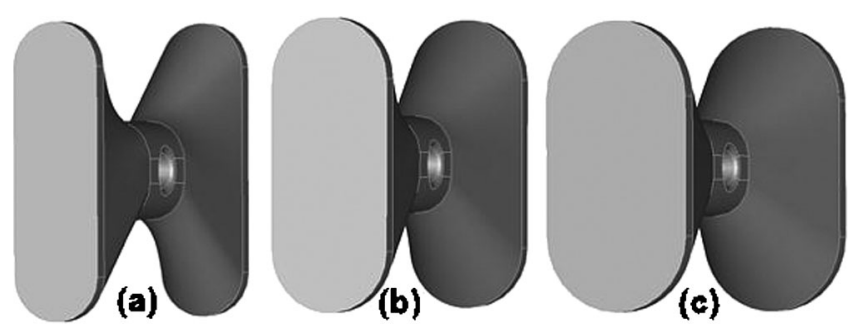

FIG. 11. Different transverse spoke base shapes with different ratios of width to length. (a) 0.30 , (b) 0.42 , and (c) 0.52 .

more desirable, then shape (a) from Fig. 11 is preferable while giving the lowest energy content. On the other hand, if lower fields are the main goal, then perhaps a shape more like (b) in Fig. 11 is a better choice.

A larger base does increase the fundamental frequency, which means that the cavity radius has to be increased to compensate. This is illustrated in Fig. 14. For the dimensions given in this example, the cylindrical geometry gives a radius of roughly $0.6 \beta_{0} \lambda$. When increasing the base length, the cavity diameter required to maintain a
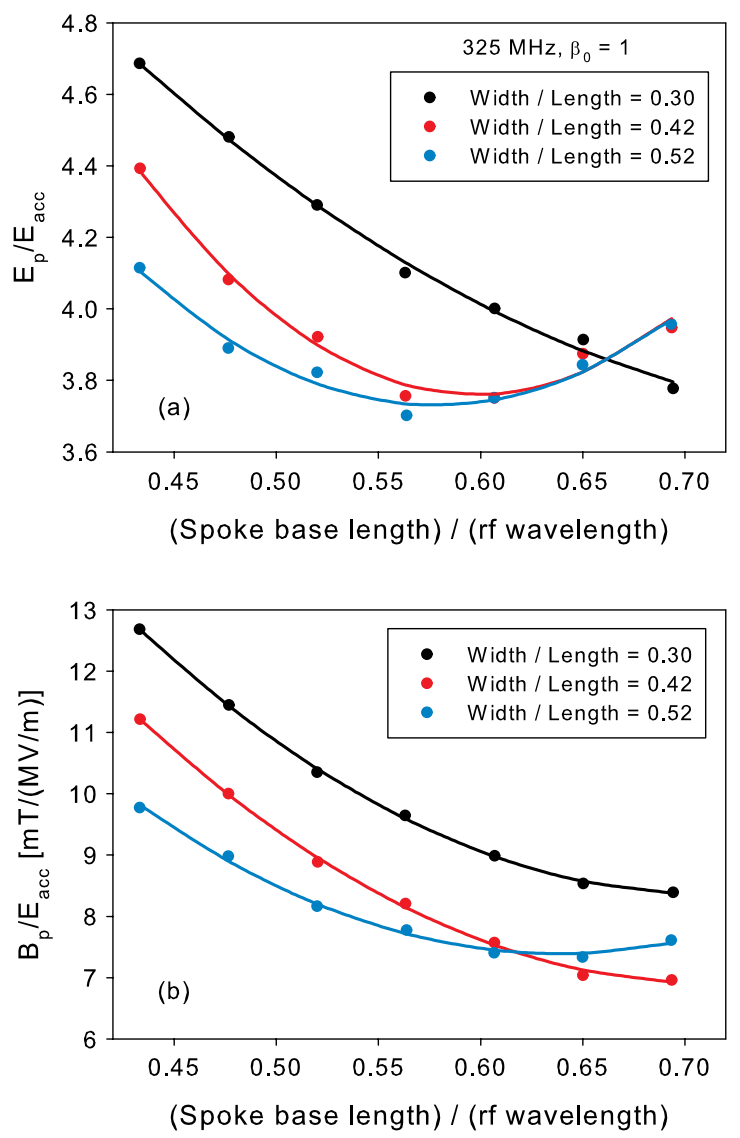

FIG. 12. Dependence of (a) $E_{p} / E_{\text {acc }}$ and (b) $B_{p} / E_{\text {acc }}$ on spoke base size. Three different transverse spoke base shapes are presented, identified by the ratio of base width to length. For each data point, both the base width and length are changing, but the ratio remains fixed.
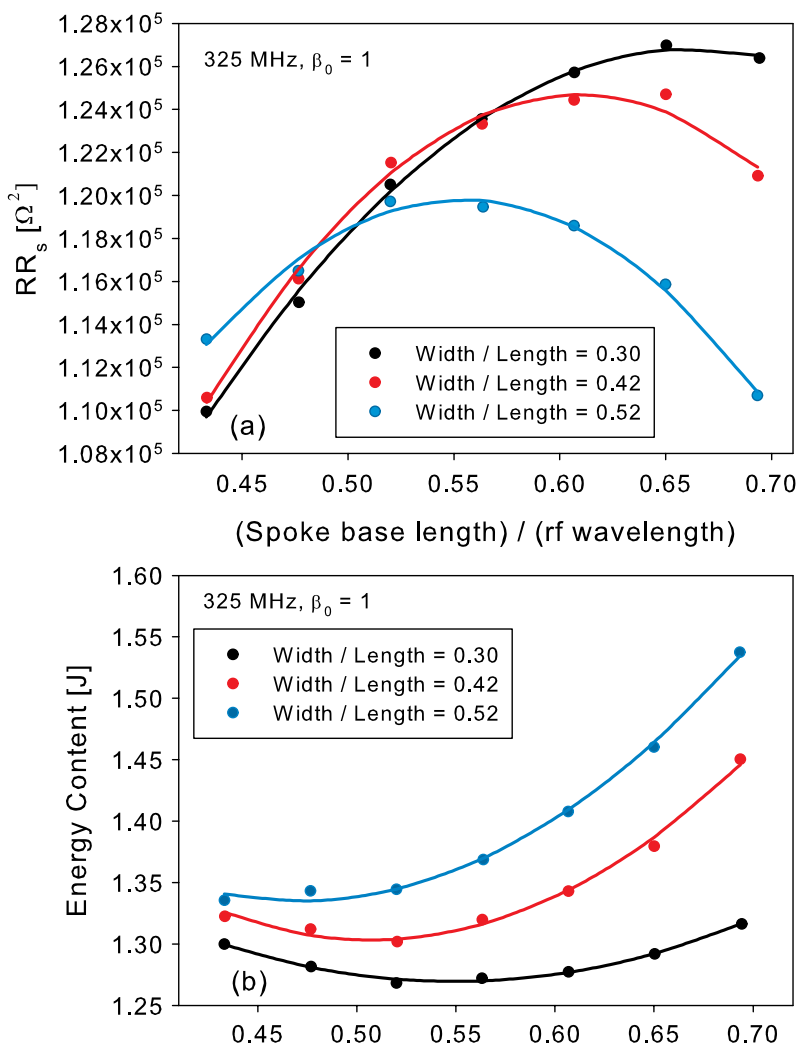

(Spoke base length) / ( $\mathrm{rf}$ wavelength)

FIG. 13. Dependence of (a) $R R_{s}$ and (b) energy content (at $1 \mathrm{MV} / \mathrm{m}$ ) on spoke base size. Three different transverse spoke base shapes are presented, identified by the ratio of base width to length. For each data point, both the base width and length are changing, but the ratio remains fixed.

frequency of $325 \mathrm{MHz}$ increases by $15 \%$ for a transverse spoke base and $10 \%$ for a longitudinal one. This can lessen the advantage spoke cavities provide in terms of compactness. Additionally, for a cylindrically shaped outer conductor, a spoke base length which approaches the diameter of the cavity itself will become increasingly difficult to fabricate and clean.

Some generalizations can be made concerning the spoke base geometry. (i) Cylindrical spoke geometries have higher surface fields. (ii) Both racetrack and elliptical geometries allow for similar peak surface fields and shunt impedance. (iii) Longitudinally oriented spoke bases, whether elliptical or racetrack, result in lower shunt impedance than that achievable with a transverse orientation. (iv) A transversely oriented spoke base allows for lower peak surface fields at the cost of increased cavity diameter. (v) Larger spoke base length and width result in a larger cavity diameter to maintain the fundamental mode frequency (Fig. 14). (vi) Transverse spoke base geometries, especially with large length, can be more difficult than their longitudinal counterparts to weld into the outer conductor. In addition, the cleaning of the cavity interior after the spokes are welded into place could prove more difficult. 


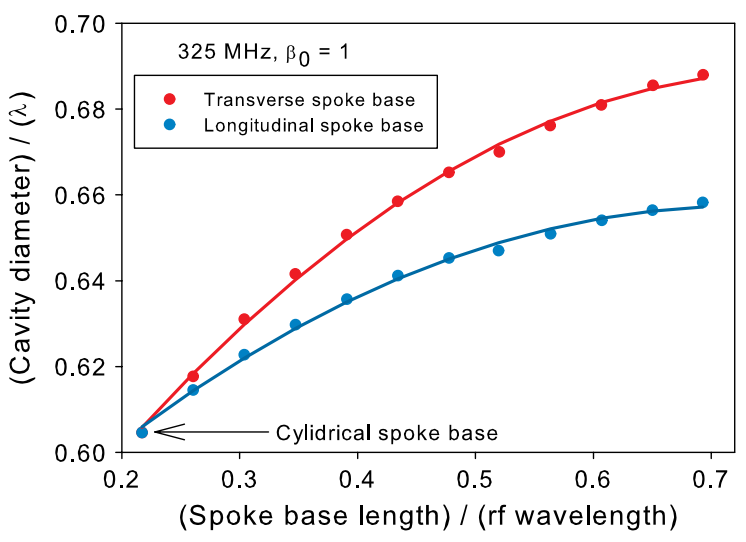

FIG. 14. Dependence of the cavity diameter on the spoke base length. A cylindrical base has the highest surface fields, but the smallest cavity diameter.

\section{Spoke aperture}

The electric field of the fundamental accelerating mode of a spoke cavity is concentrated at the accelerating gaps along the beam path. The shape and dimensions of the spoke aperture region thus have a great impact on the peak surface electric field. Because the field is concentrated in this region, achieving a uniform distribution along the surface of the spoke aperture is important for realizing a low peak surface electric field.

A similar approach has been used in optimizing the aperture region to that used in optimizing the base. We first begin by analyzing how individual parameters influence the fields and shunt impedance, then study different aperture shapes and determine the optimal size of those shapes. For this optimization, we have looked at elliptical, racetrack, and cylindrical shapes. The elliptical cross section and the racetrack are similar enough that we need not present results for both.

Figure 15(a) is an example of how the normalized electric and magnetic fields behave with respect to the spoke aperture length (both have a transverse orientation). The aperture length can be used to change the normalized electric field by $10 \%$, or more, while the normalized magnetic field can be influenced by roughly half that.

Figure 15(b) shows the dependence of $R R_{s}$ and the energy content for the same aperture dimensions. The shunt impedance is decreased (increasing the power dissipation) by around 5\% while the energy content is increased by a similar amount. Both of these results have a negative impact on the cavity performance. However, from Fig. 15, at an aperture length to rf wavelength ratio of around 0.20 , the normalized electric field is at a minimum while the adverse effects on the other rf parameters are minimal.

The dependence of these same properties on the spoke aperture width are examined in Fig. 16.

The aperture width seems to have a greater effect on the rf properties than the length. When the width of the
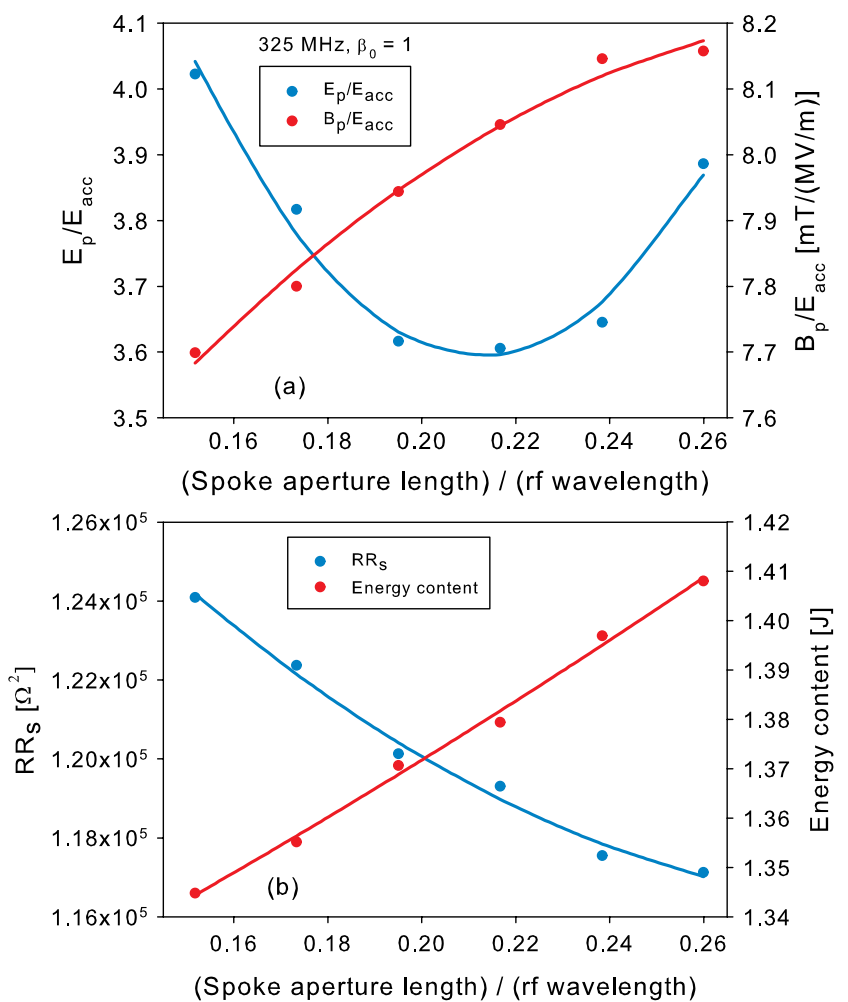

FIG. 15. (a) Dependence of the normalized electric and magnetic fields and (b) $R R_{s}$ and energy content (at $1 \mathrm{MV} / \mathrm{m}$ ) on the spoke aperture length.
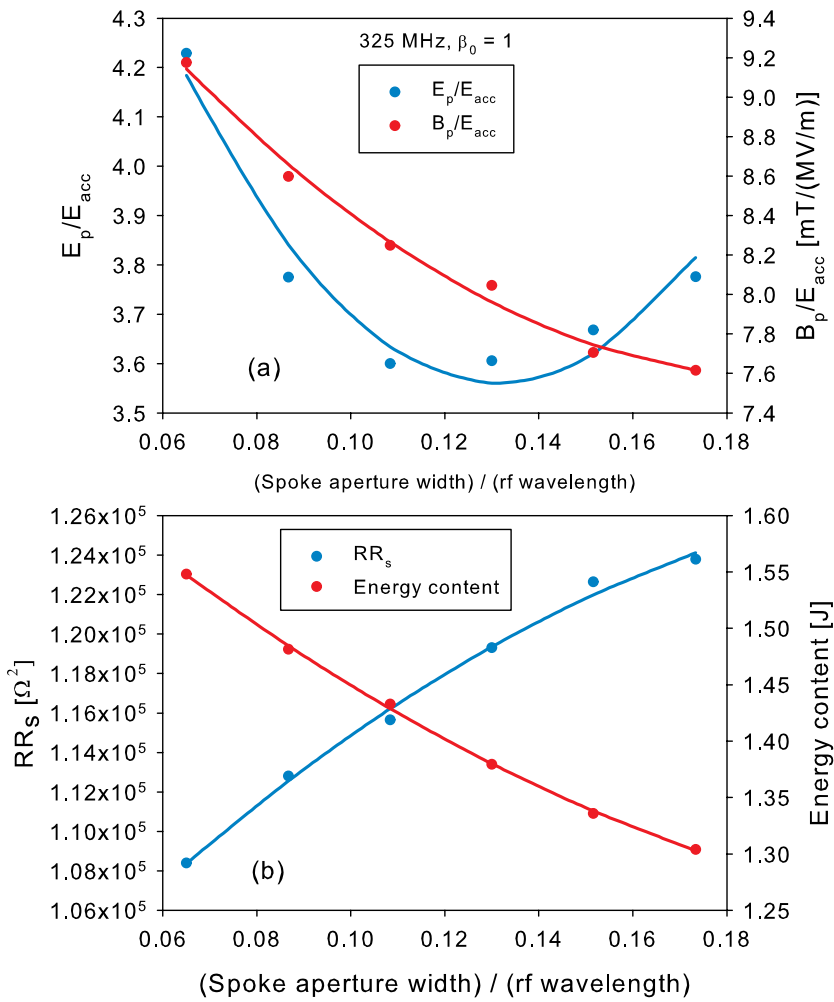

FIG. 16. Dependence of (a) $E_{p} / E_{\text {acc }}$ and $B_{p} / E_{\text {acc }}$ and (b) $R R_{s}$ and energy content (at $1 \mathrm{MV} / \mathrm{m}$ ), on the spoke aperture width. 
aperture increases, the length of each accelerating gap decreases. This has the effect of increasing the electric field for a given voltage. By doing so, there is a point for which the peak surface field will begin to increase, which is what is observed in Fig. 16. Both normalized fields can be reduced significantly, while the shunt impedance and energy content are increased and decreased, respectively.

Interestingly, the aperture height has more of an influence on the peak surface magnetic field than the electric field. This is evident in Fig. 17, as the normalized electric field changes by only a few percent while the magnetic field can increase by $20 \%$ or more as the height gets larger.

A longitudinally oriented spoke aperture produces significantly higher peak electric fields than a transverse one, thus that geometry is not discussed in detail here.

To determine what spoke aperture shapes to pursue, a plot of $B_{p}$ vs $E_{p}$ for various shapes can be seen in Fig. 18. Those designs which fall close to the lines representing $B_{p} / E_{p}=2,2.3 \mathrm{mT} /(\mathrm{MV} / \mathrm{m})$ should be optimized further to determine the shape which provides the lowest normalized fields and highest shunt impedance.

Continuing with the aperture dimensions, similar analysis to that in Figs. 12 and 13 can be done. Figure 19 first examines the normalized electric and magnetic fields for four examples of aperture shape. Here, a racetrack geometry was used for both the base and aperture regions.

Figure 19(a) illustrates that, as the spoke aperture region becomes more cylindrical, the normalized electric field

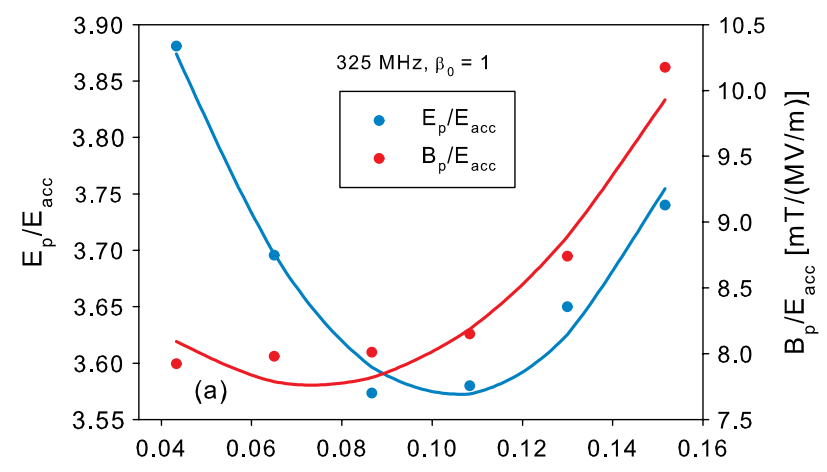

(Spoke aperture height) / (rf wavelength)

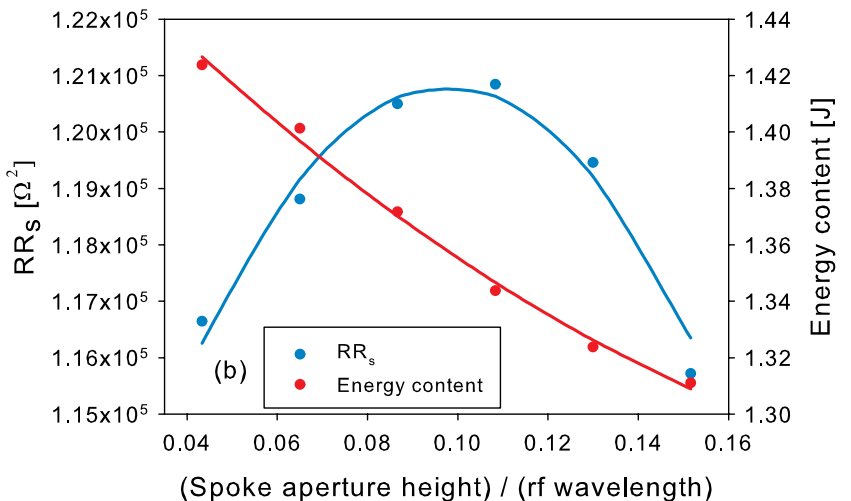

FIG. 17. Dependence of (a) $E_{p} / E_{\text {acc }}$ and $B_{p} / E_{\text {acc }}$ and (b) $R R_{s}$ and energy content (at $1 \mathrm{MV} / \mathrm{m}$ ) on spoke aperture height.

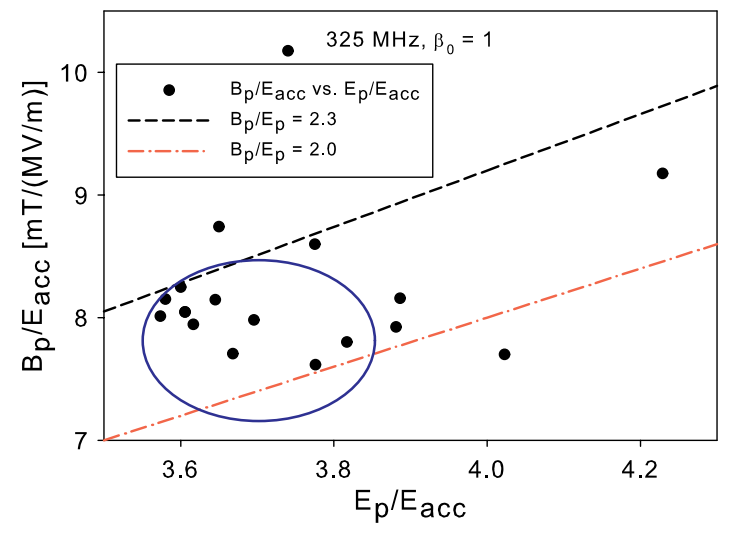

FIG. 18. Peak magnetic field vs peak electric field for various spoke aperture shapes.

achievable is higher. This can be understood in terms of the importance of creating a uniform electric field around the aperture region. Because the field is concentrated along the beam line, a cylindrically shaped aperture gives a less uniform distribution than the flat area of a transversely oriented racetrack or elliptical shape. A cylinder gives less area for which the field is in contact than a flatter, racetrack shape. The normalized magnetic field, on the
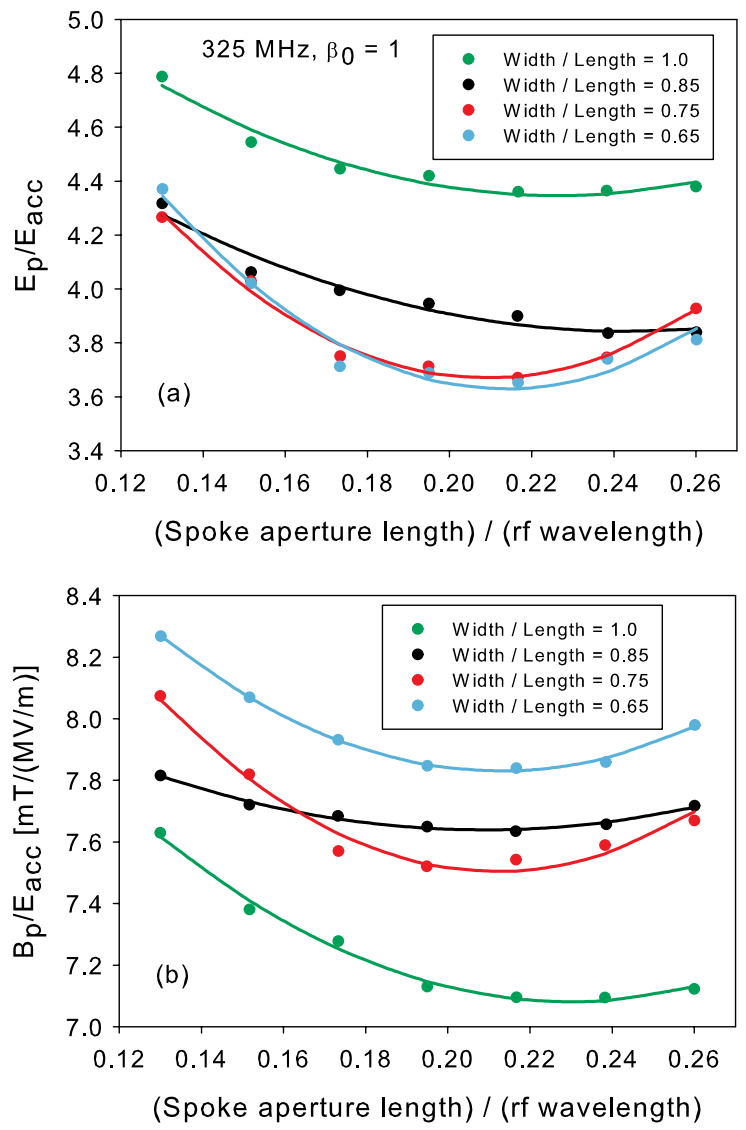

FIG. 19. Dependence of the (a) normalized electric and (b) magnetic fields on spoke aperture shapes. 


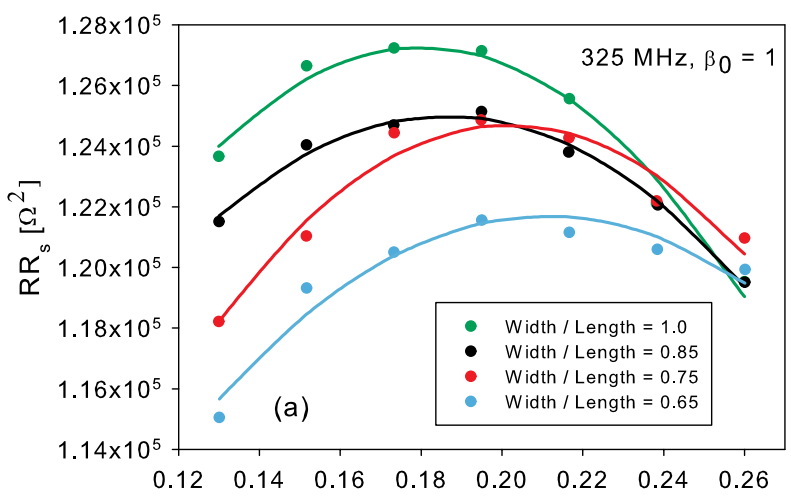

(Spoke aperture length) / ( $\mathrm{rf}$ wavelength)

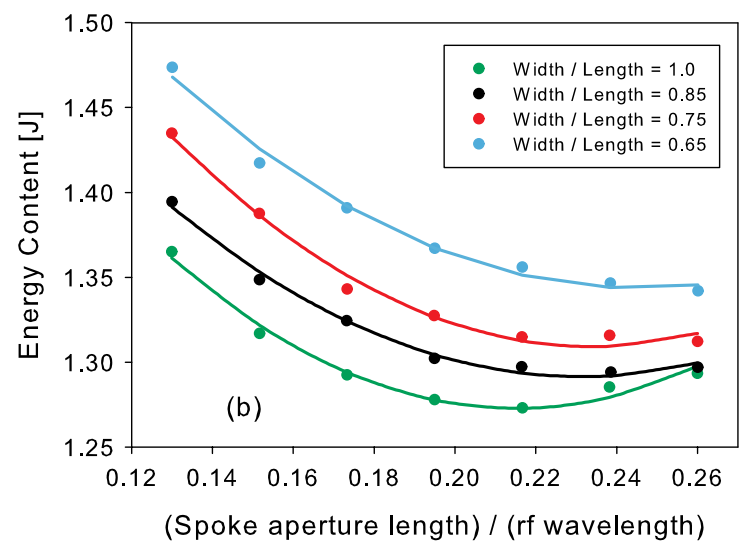

FIG. 20. Dependence of (a) $R R_{s}$ and (b) energy content (at $1 \mathrm{MV} / \mathrm{m}$ ) on spoke aperture shapes.

other hand, improves as the shape goes from more elongated (ratio of 0.65) to cylindrical.

Figure 20 shows that the shunt impedance of these shapes behaves similarly. The smaller ratio (more elongated) aperture has a lower achievable shunt impedance, albeit not much less, than the other shapes. A cylindrical aperture region allows for close to a 5\% higher shunt impedance than the more elongated racetrack shape. It is not surprising that the smaller ratio then has a higher energy content, since it is inversely proportional to the shunt impedance.

To summarize these results, a cylindrical aperture region gives a lower normalized magnetic field, higher shunt impedance, and lower energy content. An elongated racetrack-shaped aperture region gives the lowest normalized electric field. A racetrack shape with a ratio of width to length somewhere between 0.65 and 1.0 may be preferable for most applications.

Recently, there have been efforts made to decrease the quadrupole component by using a ring-shaped aperture region [37] in a half-wave resonator. It was found that, in addition to achieving this, the peak surface magnetic field and shunt impedance could also be improved with this geometry. We have investigated this geometry (Fig. 21) and found that it does indeed reduce the quadrupole

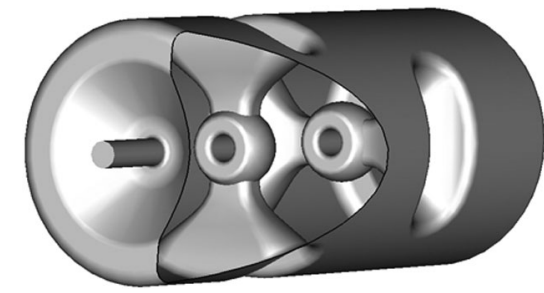

FIG. 21. Design of a ring-shaped aperture we are using to study possible reduction in multipole effects. This design is adapted from [37].

component of the off-axis electromagnetic field, but we did not see significant improvements in the peak surface fields or shunt impedance over what has been reported here. This component of the field was reported for our $325 \mathrm{MHz}, \beta_{0}=0.82$ cavity in [38]. A more detailed analysis of the multipole components for this cavity and how different geometries affect those components has been presented elsewhere [39].

\section{Spoke separation}

In low- $\beta_{0}$ structures made with several loading elements (multispoke, split ring, or twin quarter wave, for example), the side gaps are approximately half the size of the central gap and the energy gained in the side gaps is approximately half that gained in the central gaps. As was already noted, the distance between the centers of the side gaps, to a large degree, determines the $\beta_{0}$ of a spoke cavity. Therefore, for high- $\beta_{0}$ cavities at the frequencies discussed here, the cavity length becomes greater than a meter. This large size can add some additional challenges, however there are some advantages to be gained. This longer cavity provides a great deal of flexibility in the spoke base dimensions.

Figure 22 shows what is referred to here as the spoke angle $\phi$, which is a measure of the difference between the transversely oriented spoke aperture and base length and

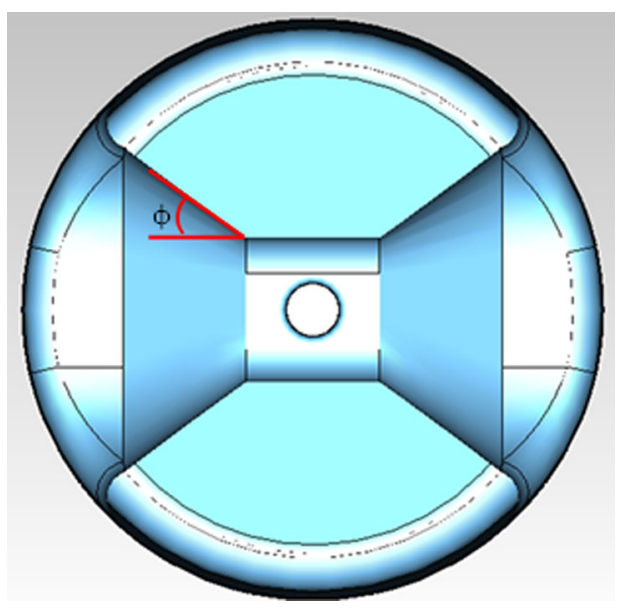

FIG. 22. End view of a single spoke showing the spoke angle $\phi$. 
height. This angle not only has a strong effect on the surface fields, but also on the spoke separation. Balancing the fields in the accelerating gaps of a multispoke resonator is not paramount, however $E_{p} / E_{\text {acc }}$ will be approximately minimized when the electric field is homogeneously distributed on the spoke surfaces. If, during a sweep of the parameter $\phi$, the spoke separation remains constant along with $\beta_{0}$ and frequency, the distribution of the electric field on the beam line will become severely inhomogeneous, causing the normalized electric field to increase. If the length of the spoke aperture region is on the order of those in Fig. $22\left(\sim 0.10 \beta_{0} \lambda\right)$, then for a small $\phi$, the spoke separation is very close to one-half of the total iris-to-iris distance. On the other hand, as $\phi$ becomes larger for a transversely oriented spoke base, the structure begins to resemble a disk-loaded waveguide, and as such, the spoke separation required to balance the fields in each cell becomes close to the distance between each spoke and the end walls (one-third of the iris-to-iris length). Figure 23 shows how the electric field profiles change for different values of $\phi$ if the electric fields in each cell are equalized by changing the spoke separation. For the small spoke angle $\left(20.5^{\circ}\right)$, the center gap is approximately $\beta_{0} \lambda / 2$ whereas for $\phi=44.4^{\circ}$, the gaps are roughly the same size.

Figure 24 shows the dependence of the normalized electric and magnetic fields on spoke separation. We use units of $\beta_{0} \lambda / 2$ because in a cavity with more uniform spokes, that would be a typical spoke separation.

The effect that the spoke separation can have on the cavity properties obviously depends, to a large degree, on the spoke base and aperture dimensions, so we will not attempt to generalize these effects, but will summarize the effects that the spoke separation has on one of our optimized cavities ( $\left.325 \mathrm{MHz}, \beta_{0}=1\right)$. These data are taken with a spoke angle of roughly $40^{\circ}$, so when the normalized electric field is lowest, the field is approximately uniform in each gap. This occurs at a spoke separation of $\sim 0.84$ $\beta_{0} \lambda / 2$. At that point, the normalized magnetic field is significantly greater than its lowest value. In order to

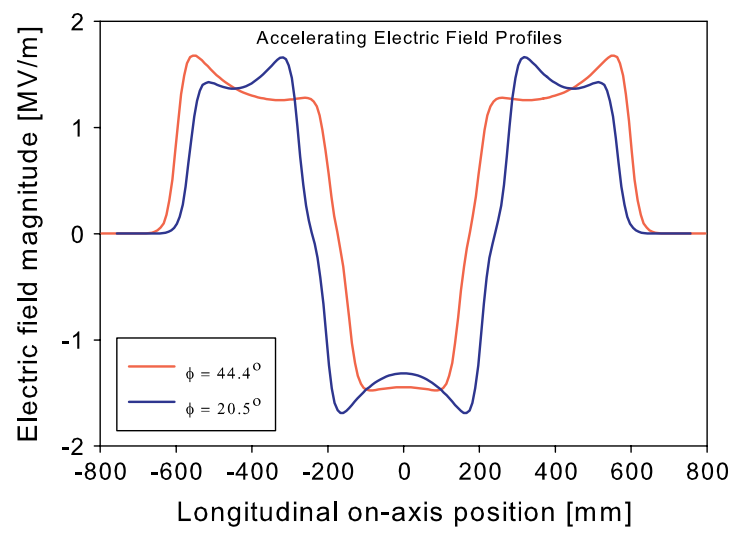

FIG. 23. On-axis electric field profiles (at $1 \mathrm{~J}$ ) for different spoke angle $\phi$.

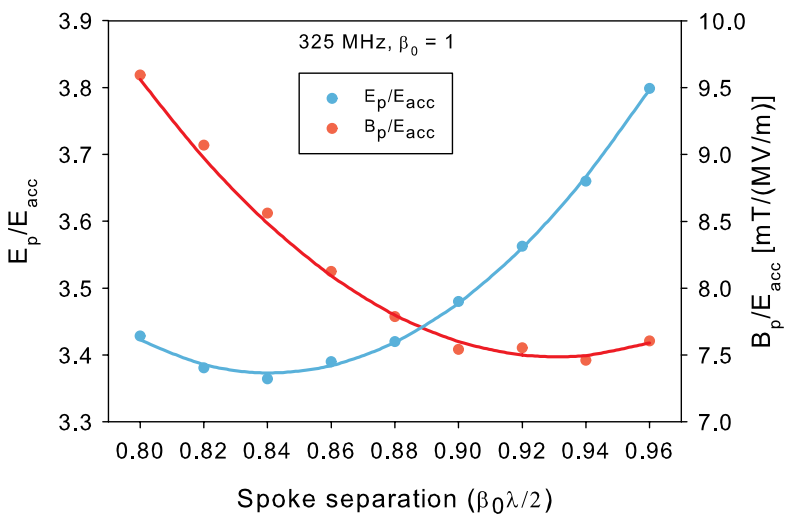

FIG. 24. Dependence of the normalized electric and magnetic fields on spoke separation, for a spoke angle of $36^{\circ}$.

balance the normalized fields, a spoke separation of around $0.94 \beta_{0} \lambda / 2$ would be a good choice for this example.

The dependence the shunt impedance has on the spoke separation is shown in Fig. 25. Not surprisingly, when the normalized magnetic field is lowest, the power dissipation is also low, and thus the shunt impedance is highest.

Finally, it should be noted that when the spoke angle is large and the spokes are separated to minimize the normalized magnetic field, the velocity acceptance of the cavity decreases, as shown in Fig. 26. This is less of a concern for velocity-of-light cavities, but should be considered for cavities with a design velocity less than 1 .

To conclude this section, we summarize some observations made concerning the spoke separation of the $325 \mathrm{MHz}, \beta_{0}=1$ cavity: (i) The normalized electric field is approximately minimized when the field is distributed homogeneously on the spoke surface in the accelerating region. (ii) The optimal spoke separation depends greatly on $\phi$. For fixed aperture dimensions, as $\phi$ increases, the spoke separation needed to minimize the normalized electric field decreases. (iii) Increasing the spoke separation beyond the point at which the magnitude of the electric field is equal in each accelerating gap reduces the velocity acceptance of the cavity.

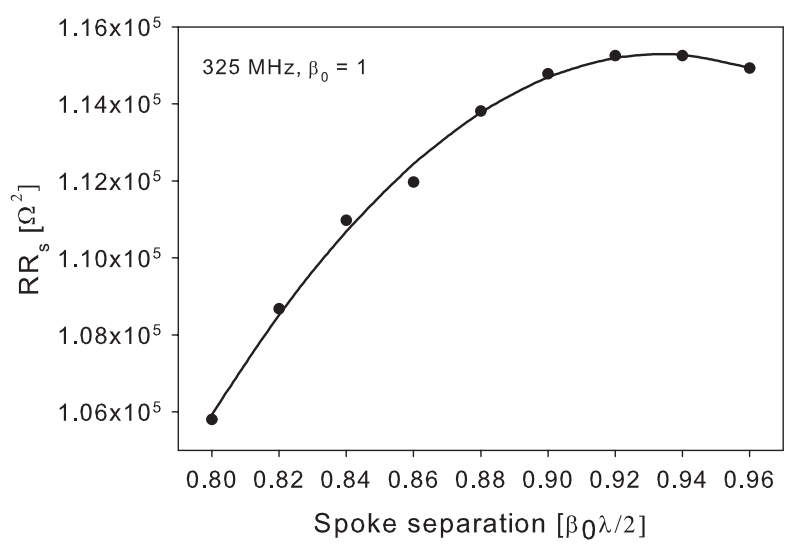

FIG. 25. Dependence of $R R_{s}$ on spoke separation. 


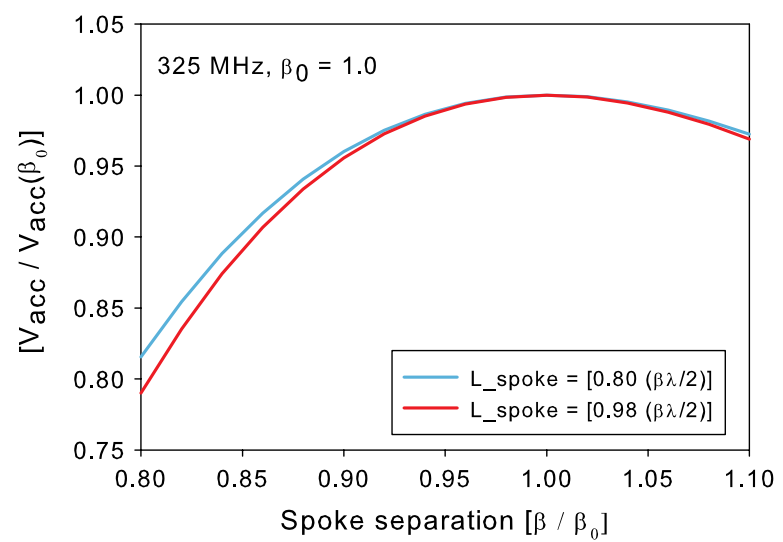

FIG. 26. Velocity acceptance for two different spoke separations.

\section{Reentrant parameters $\left(h \_o u t, h \_i n\right.$, and $\left.H\right)$}

Referring to Fig. 4, h_out can be used to minimize the peak surface magnetic field, $h$ _in can be used to minimize the peak surface electric field, and $\mathrm{H}$ will strongly influence both. As with optimization of any parameter with respect to the peak surface fields, it is important to also consider maximizing the shunt impedance. In the case of parameters such as these, which do not impact the surface fields as greatly as the spoke parameters, we find that there can be a small gain in shunt impedance while obtaining a mild reduction in peak surface fields. Figure 27 shows how h_out impacts both the normalized magnetic field and $R R_{s}$.

It is clear that increasing h_out has positive effects on the normalized magnetic field by increasing the volume for which the field has to encircle the spokes. This also results in a decreased power dissipation which leads to a higher shunt impedance.

$\mathrm{H}$ is a parameter, as can be seen in Fig. 4, which has an effect on both the normalized magnetic and electric fields. Figure 28 shows how the normalized magnetic field and shunt impedance change for a varying $\mathrm{H}$, while Fig. 29 shows how the normalized electric field and shunt impedance change.

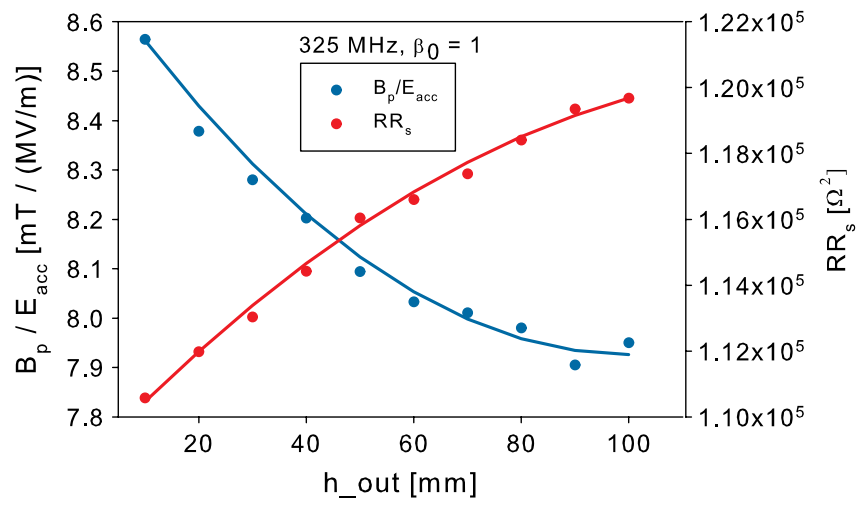

FIG. 27. Dependence of the normalized magnetic field and $R R_{s}$ on the parameter h_out.

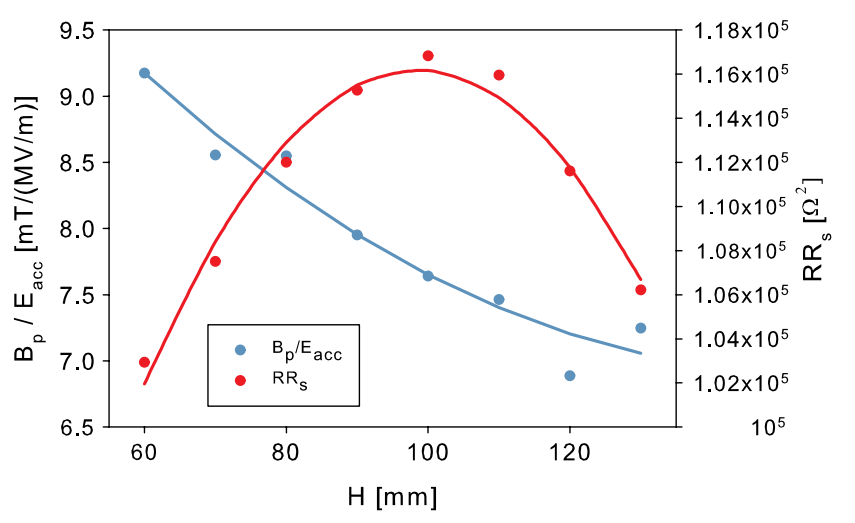

FIG. 28. Dependence of the normalized magnetic field and $R R_{s}$ on the parameter $\mathrm{H}$.

The parameter $\mathrm{H}$ changes the overall length of the cavity, but does not change the iris-to-iris distance. In Fig. 28 it is clear that as this parameter increases, the normalized surface magnetic field decreases. The opposite is actually true for the normalized electric field as can be seen in Fig. 29. Thus, in the cavities examined here, there is no single value of $H$ that can minimize either of the fields while simultaneously maximizing the shunt impedance, but from Figs. 28 and 29, a value of around $100 \mathrm{~mm}$, for this $325 \mathrm{MHz}, \beta_{0}=1$ double-spoke cavity, sufficiently minimizes both fields while maximizing the shunt impedance.

Finally, since $\mathrm{h}$ _in is a parameter which is close to the beam line, it can be used to optimize the normalized electric field. Figure 30 shows how the normalized electric field and shunt impedance change with varying $h \_i n$.

\section{Curve rounding}

Another consideration with regard to field optimization is the rounding radius applied to otherwise sharp edges formed when designing the cavity. There are a few areas of the cavity where the rounding radius can have a significant affect on the fields, two of which are discussed here and shown in Fig. 31.

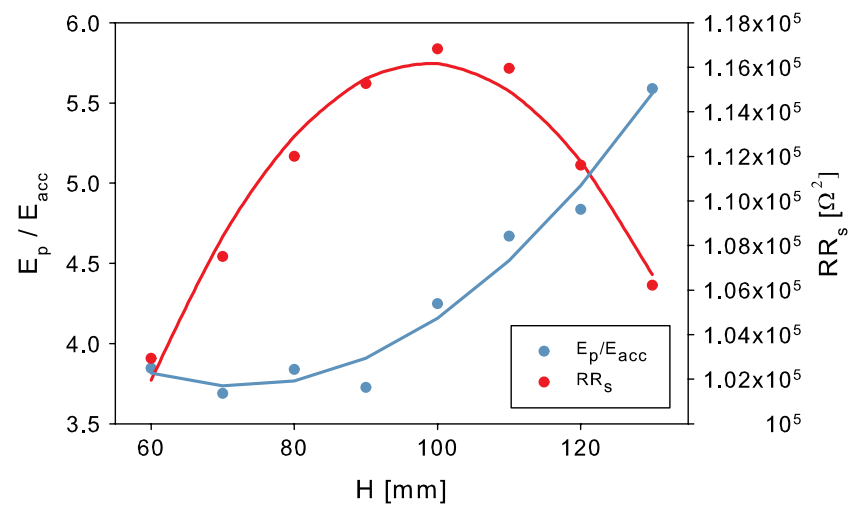

FIG. 29. Dependence of the normalized electric field and $R R_{s}$ on the parameter $\mathrm{H}$. 


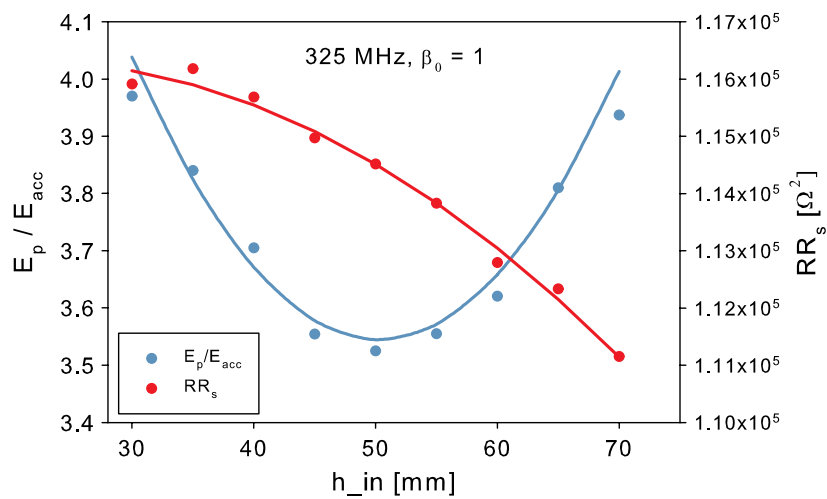

FIG. 30. Dependence of the normalized electric field and $R R_{s}$ on the parameter $h \_$in.

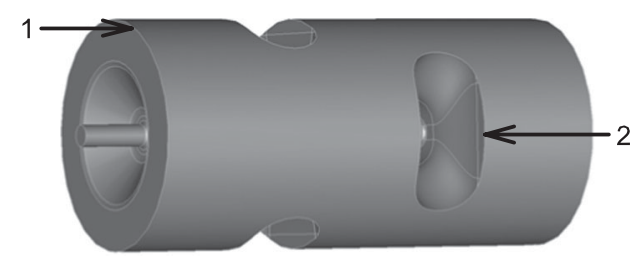

FIG. 31. Cavity with no rounding at (1) the outer conductor edges and (2) the spoke base.

In Fig. 32, we can see how the normalized magnetic field changes for a wide range of outer conductor edge rounding. There is almost a $10 \%$ difference in the two extremes.

The spoke base rounding radius (identified as " 2 " in Fig. 31) also has a strong influence on the peak surface magnetic field, as Fig. 33 illustrates.

Increasing the spoke base rounding radius decreases the normalized magnetic field, while there is a large range of outer conductor rounding radii for which the peak magnetic field is virtually unchanged.

When determining an optimal rounding, the impact on multipacting should be considered. While the effect cannot be eliminated completely because it is much more

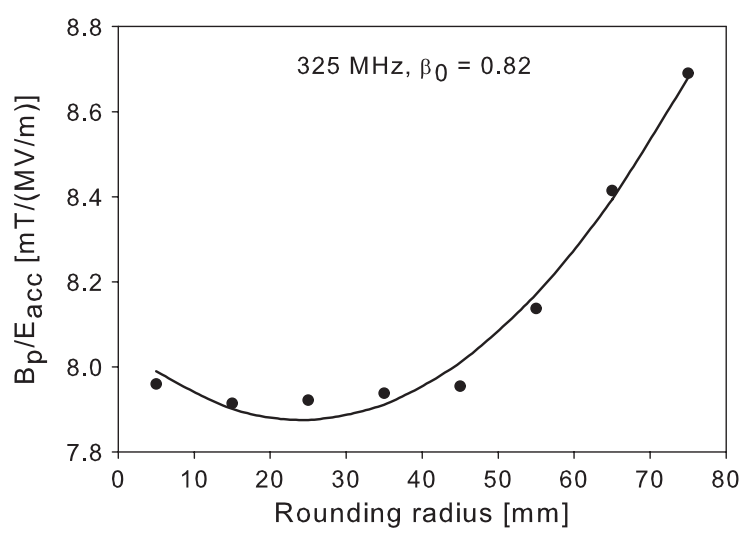

FIG. 32. Dependence of $B_{p} / E_{\text {acc }}$ on the outer conductor edge rounding.

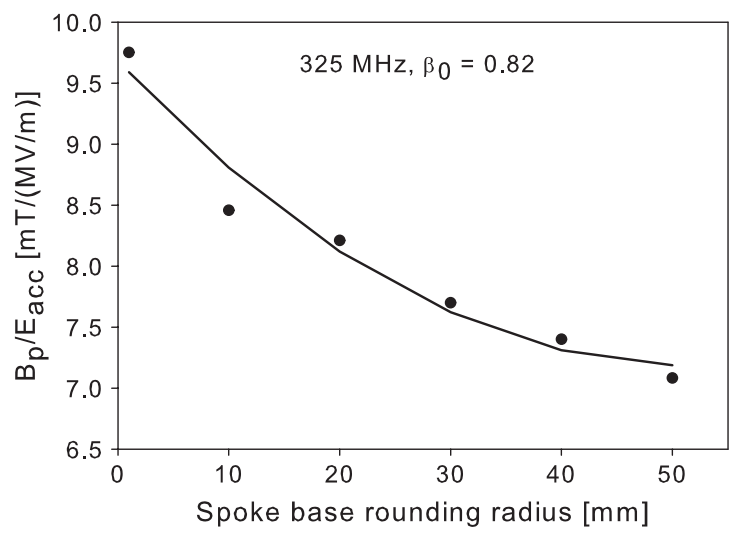

FIG. 33. Dependence of $B_{p} / E_{\text {acc }}$ on the spoke base rounding radius.

dependent on the cavity surface conditions and ability to provide adjustable levels of power to the susceptible regions of the cavity, we have found that it can be reduced through simple modifications to the geometry.

When the internal surface of a rf cavity is exposed to the high fields maintained in a superconducting cavity, electrons (known as primary electrons) can be emitted from the metal. The trajectory of these electrons is determined by the electromagnetic fields, and in many cases, they will come in contact with another part of the surface with a certain amount of impact kinetic energy. If, for relatively clean niobium, this energy falls within the range of $\sim 100 \mathrm{eV}$ to $1500 \mathrm{eV}$, then additional electrons, known as secondary electrons, will be ejected, which is referred to as secondary emission yield (SEY) [40]. These electrons can even end up in resonant trajectories, at which point this multipacting process can generate a large amount of excess heat, thus leading to thermal breakdown.

Additionally, these secondary electrons will absorb energy from the rf fields, thereby limiting the gradient which would otherwise be achievable in their absence. The SEY is not only material specific, but also depends of the condition of the surface [40]. By improving the quality of the surface, the soft barriers on the gradient can be eliminated by processing and cleaning. On the other hand, hard barriers can only be overcome by changing the cavity geometry.

Multipacting is characterized, most commonly, as either one-point or two-point. One-point multipacting occurs when the time of flight of the electron between two impacts is an integer number of rf cycles and the electron's impact site is approximately the same as its ejection site. In twopoint multipacting, the time of flight is an odd number of half rf cycles and the impact site is not the same as the ejection site.

In order to analyze the multipacting conditions in these optimized two-spoke cavities, the 3D parallel tracking code TRACK3P contained in the ACE3P code suite developed by SLAC was used [41]. In Fig. 34, we show some resonant 


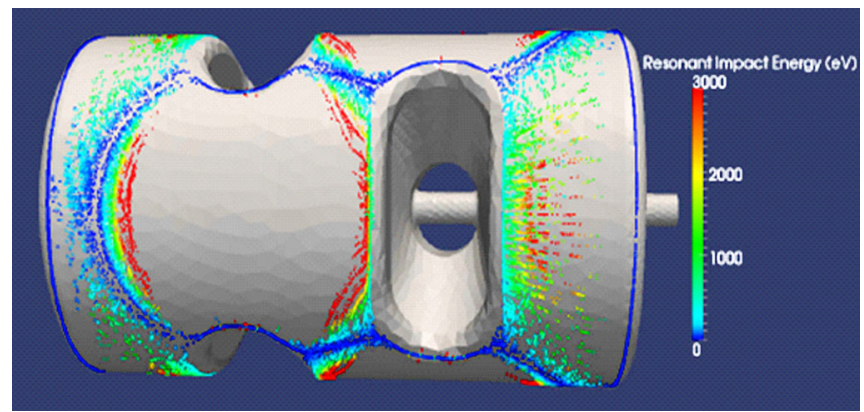

FIG. 34. Resonant electrons and their energies for field levels from $0.5-10 \mathrm{MV}$.

electrons for field gradient levels from 0.5 to $10 \mathrm{MV} / \mathrm{m}$ in an optimized $325 \mathrm{MHz}, \beta_{0}=0.82$ cavity. The impact energy is also shown.

Many of the areas where SEY would be likely are around where the spoke base meets the outer conductor and at the end cap, so these rounding radii can have an impact on the number of electron that fall into resonant trajectories [42]. This cannot be avoided, but slightly altering the geometry to minimize multipacting should be considered.

To address possible soft barriers at high field gradient, the inset of Fig. 35 shows the multipacting electron energies present from 6-10 MV/m for this region of the cavity. The plots are for two different rounding radii at the outer edge. A more subtle rounding (shown in black) has electrons with resonant impact energies of up to about $250 \mathrm{eV}$, although most fall below $150 \mathrm{eV}$. On the other hand, a larger rounding radius (shown in red) results in virtually all of the electron energies being below $100 \mathrm{eV}$. Very similar electron trajectories are also stable at the rounded area where the spoke meets the outer conductor. As such, that

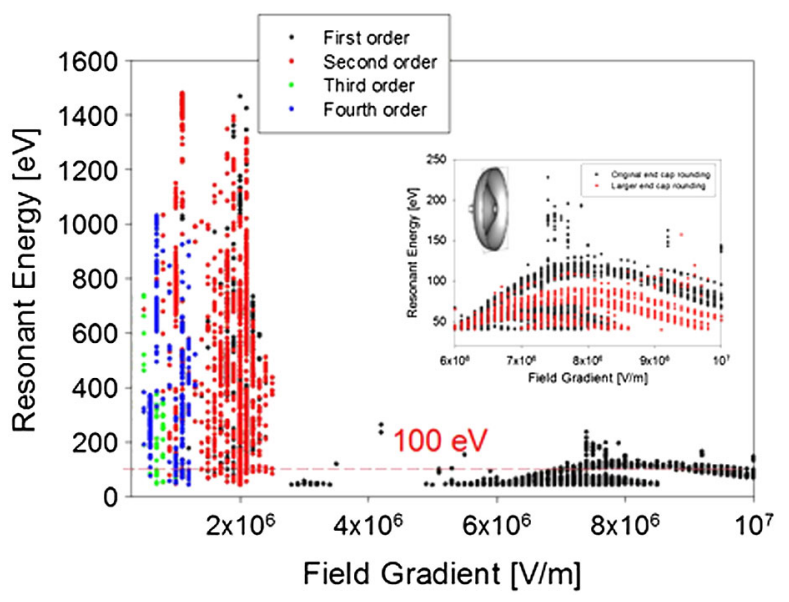

FIG. 35. Resonant electron impact energy in an end cell of the cavity in Fig. 34 for gradients up to $10 \mathrm{MV} / \mathrm{m}$. The inset shows the impact energies for stable multipacting electrons for two different end-cap outer rounding radii. rounding radius can be used to minimize the multipacting in that area as well.

\section{RESULTS}

\section{A. Field optimization}

The $\beta_{0}=0.82$ models presented here have been designed with applications to high-energy protons and ions in mind, and as such, an important optimization consideration is to reduce the peak surface magnetic and electric fields. The preceding sections provide a detailed discussion of how the most influential parameters affect the fields and shunt impedance, and in this section we present the results of field minimization optimizations.

Before doing so, there is another consideration to be made, namely, the placement of the coupler ports. One advantage of spoke cavities, which was mentioned previously, is that couplers can be placed on the outer conductor. Although not reported here, we have included a fundamental power coupler port, cleaning ports (shown in Fig. 1), and higher order mode damping ports in simulations of the final design, and have found that through judicious placement, the electromagnetic properties are not negatively affected.

Figure 36 shows the surface electric and magnetic fields for the optimized $325 \mathrm{MHz}, \beta_{0}=0.82$ cavity. Tables I, II, III, and IV provide the cavity parameters and rf properties of four high- $\beta_{0}$ cavities that have been optimized to date. The aperture diameter was fixed at $60 \mathrm{~mm}$ for the $325 \mathrm{MHz}$ cavities and $50 \mathrm{~mm}$ for the $352 \mathrm{MHz}$ cavities. The actual cavities which will be built and tested may be somewhat different based on fabrication and ease of cleaning considerations.

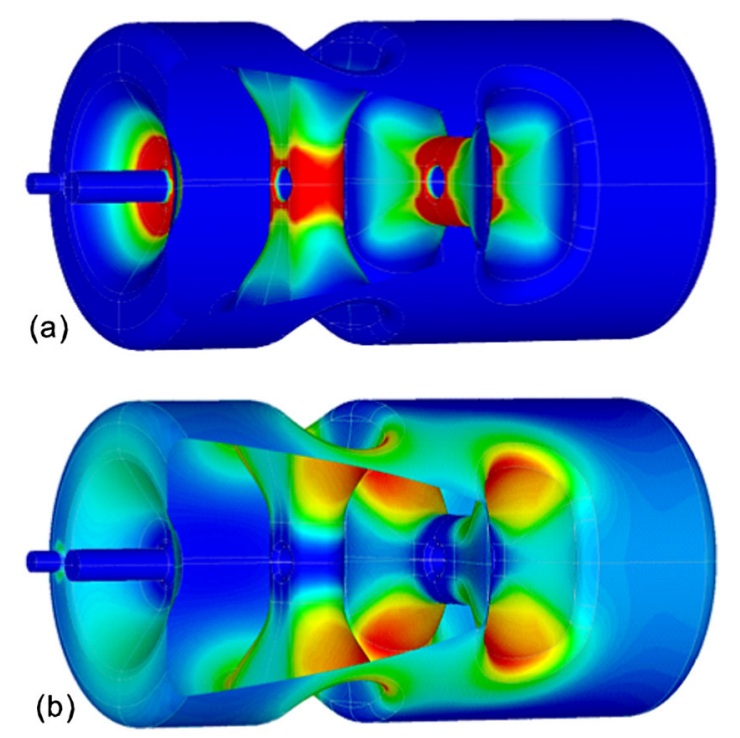

FIG. 36. CST MWS (C) cut-away view showing the surface (a) electric and (b) magnetic fields of the fundamental mode of the optimized $325 \mathrm{MHz}, \beta_{0}=0.82$ cavity. 
TABLE I. Cavity parameters, $352 \mathrm{MHz}, \beta_{0}=0.82$ and $\beta_{0}=1$.

\begin{tabular}{lcrc}
\hline \hline Parameter & $\beta_{0}=0.82$ & $\beta_{0}=1$ & Units \\
\hline Frequency of accelerating mode & 352 & 352 & $\mathrm{MHz}$ \\
Frequency of nearest mode & 360 & 358 & $\mathrm{MHz}$ \\
Cavity diameter & 560 & 585 & $\mathrm{~mm}$ \\
Iris-to-iris length & 859 & 1061 & $\mathrm{~mm}$ \\
Cavity length & 1042 & 1226 & $\mathrm{~mm}$ \\
Reference length $\left[(3 / 2) \beta_{0} \lambda\right]$ & 1048 & 1278 & $\mathrm{~mm}$ \\
Aperture diameter & 50 & 50 & $\mathrm{~mm}$ \\
\hline \hline
\end{tabular}

TABLE II. Radio-frequency properties, $352 \mathrm{MHz}, \beta_{0}=0.82$ and $\beta_{0}=1$.

\begin{tabular}{lccc}
\hline \hline Parameter & $\beta_{0}=0.82$ & $\beta_{0}=1$ & Units \\
\hline Energy gain at $\beta_{0}{ }^{\mathrm{a}}$ & 1048 & 1278 & $\mathrm{kV}$ \\
$R / Q$ & 642 & 715 & $\Omega$ \\
$Q R_{s}$ & 158 & 175 & $\Omega$ \\
$(R / Q) \cdot Q R_{s}$ & $1.01 \times 10^{5}$ & $1.25 \times 10^{5}$ & $\Omega^{2}$ \\
$E_{p} / E_{\mathrm{acc}}$ & 3.64 & 3.69 & $\cdots$ \\
$B_{p} / E_{\mathrm{acc}}$ & 7.06 & 7.06 & $\mathrm{mT} /(\mathrm{MV} / \mathrm{m})$ \\
$B_{p} / E_{p}$ & 1.94 & 1.91 & $\mathrm{mT} /(\mathrm{MV} / \mathrm{m})$ \\
Energy content $^{\mathrm{a}}$ & 0.77 & 1.03 & $\mathrm{~J}$ \\
Power dissipation $^{\mathrm{a}, \mathrm{b}}$ & 0.79 & 0.92 & $\mathrm{~W}$ \\
\hline \hline
\end{tabular}

${ }^{\mathrm{a}}$ At $E_{\mathrm{acc}}=1 \mathrm{MV} / \mathrm{m}$ and reference length $(3 / 2) \beta_{0} \lambda$.

${ }^{\mathrm{b}} R_{s}=73 \mathrm{n} \Omega$.

\section{B. Shunt impedance optimization}

One of the motivations for spoke cavity development is that they are generally compact enough to operate at lower frequencies such that they need only be cooled to $4.2 \mathrm{~K}$. For applications which require relatively small machines, especially those intended to run in continuous wave (cw) mode, operating at $2 \mathrm{~K}$ may not be feasible, therefore interest in spoke cavities operating at $\beta_{0}=1$ is growing. The optimization considerations for these velocity-of-light cavities intended to be used in compact accelerators are somewhat different than that of cavities to be used in more conventional machines. In the former, power dissipation is perhaps more important, therefore maximizing the shunt impedance is the highest priority.

From Eq. (8), it is clear that the shunt impedance is a measure of how much accelerating voltage can be gained

TABLE III. Cavity parameters, $325 \mathrm{MHz}, \beta_{0}=0.82$ and $\beta_{0}=1$.

\begin{tabular}{lccc}
\hline \hline Parameter & $\beta_{0}=0.82$ & $\beta_{0}=1$ & Units \\
\hline Frequency of accelerating mode & 325 & 325 & $\mathrm{MHz}$ \\
Frequency of nearest mode & 332 & 331 & $\mathrm{MHz}$ \\
Cavity diameter & 606 & 626 & $\mathrm{~mm}$ \\
Iris-to-iris length & 932 & 1135 & $\mathrm{~mm}$ \\
Cavity length & 1132 & 1315 & $\mathrm{~mm}$ \\
Reference length $\left[(3 / 2) \beta_{0} \lambda\right]$ & 1135 & 1384 & $\mathrm{~mm}$ \\
Aperture diameter & 60 & 60 & $\mathrm{~mm}$ \\
\hline \hline
\end{tabular}

TABLE IV. Radio-frequency properties, $325 \mathrm{MHz}, \beta_{0}=0.82$ and $\beta_{0}=1$.

\begin{tabular}{lccc}
\hline \hline Parameter & $\beta_{0}=0.82$ & $\beta_{0}=1$ & Units \\
\hline Energy gain at $\beta_{0}{ }^{\mathrm{a}}$ & 1135 & 1384 & $\mathrm{kV}$ \\
$R / Q$ & 634 & 737 & $\Omega$ \\
$Q R_{s}$ & 158 & 173 & $\Omega$ \\
$(R / Q) \cdot Q R_{s}$ & $1.00 \times 10^{5}$ & $1.28 \times 10^{5}$ & $\Omega^{2}$ \\
$E_{p} / E_{\mathrm{acc}}$ & 3.69 & 3.67 & $\cdots$ \\
$B_{p} / E_{\mathrm{acc}}$ & 7.06 & 7.06 & $\mathrm{mT} /(\mathrm{MV} / \mathrm{m})$ \\
$B_{p} / E_{p}$ & 1.91 & 1.92 & $\mathrm{mT} /(\mathrm{MV} / \mathrm{m})$ \\
Energy content $^{\mathrm{a}}$ & 0.99 & 1.27 & $\mathrm{~J}$ \\
Power dissipation $^{\mathrm{a}, \mathrm{b}}$ & 0.88 & 1.02 & $\mathrm{~W}$ \\
\hline \hline
\end{tabular}

${ }^{\mathrm{a} A t} E_{\text {acc }}=1 \mathrm{MV} / \mathrm{m}$ and reference length $(3 / 2) \beta_{0} \lambda$.

${ }^{\mathrm{b}} R_{s}=68 \mathrm{n} \Omega$.

for a given dissipation. Increasing the shunt impedance, therefore, is how the dissipation can be decreased. The power dissipation can be written as

$$
P_{d}=\frac{V_{0}^{2}}{(R / Q) \times G} \times R_{s}
$$

where the voltage, here, is normalized to an accelerating gradient of $1 \mathrm{MV} / \mathrm{m}$, reference length $(3 / 2) \beta_{0} \lambda$, and the surface resistance is fixed for a given frequency. Thus, to decrease the power dissipation, the product of $R / Q$ and $G$ should be increased. Again, both of these parameters are determined solely by the geometry of the cavity and not by the size or material properties.

In elliptical cavities, optimizing the geometry of the outer walls to maximize $Q_{0}$ often leads to a spherical shape because this corresponds to the smallest surface area for a given volume [43]. We find a similar argument can be made in the case of these particular geometries, as is illustrated in Fig. 37, where the dependence of $R R_{s}$ on the ratio of total surface area to volume (multiplied by $\mathrm{rf}$ wavelength to eliminate units) is shown. At this point, we

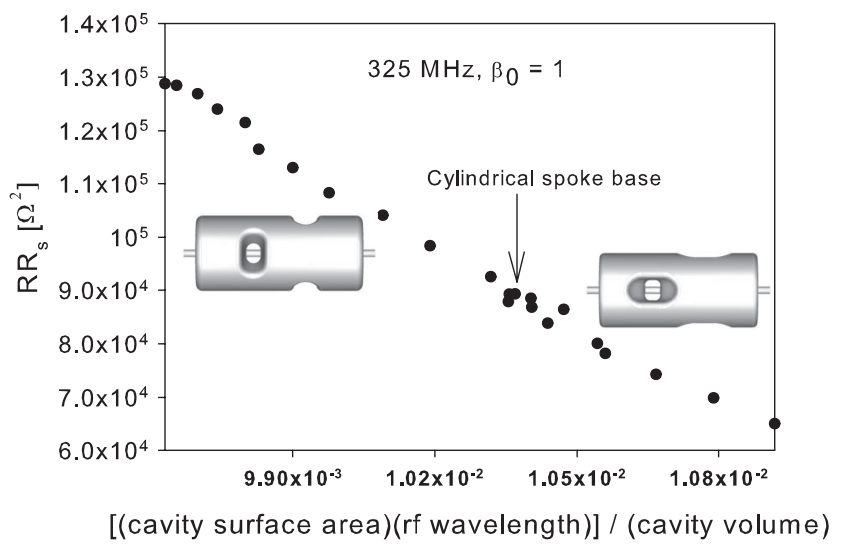

FIG. 37. Dependence of $R R_{s}$ on the ratio of cavity surface area to volume. 
TABLE V. Radio-frequency properties, $352 \mathrm{MHz}, \beta_{0}=0.82$ and $\beta_{0}=1$ cavities optimized for higher shunt impedance.

\begin{tabular}{lccc}
\hline \hline Parameter & $\beta_{0}=0.82$ & $\beta_{0}=1$ & Units \\
\hline Energy gain at $\beta_{0}{ }^{\mathrm{a}}$ & 1048 & 1278 & $\mathrm{kV}$ \\
$R / Q$ & 647 & 757 & $\Omega$ \\
$Q R_{s}$ & 173 & 180 & $\Omega$ \\
$(R / Q) \cdot Q R_{s}$ & $1.12 \times 10^{5}$ & $1.36 \times 10^{5}$ & $\Omega^{2}$ \\
$E_{p} / E_{\mathrm{acc}}$ & 4.30 & 4.05 & $\cdots$ \\
$B_{p} / E_{\mathrm{acc}}$ & 7.81 & 7.58 & $\mathrm{mT} /(\mathrm{MV} / \mathrm{m})$ \\
$B_{p} / E_{p}$ & 1.82 & 1.87 & $\mathrm{mT} /(\mathrm{MV} / \mathrm{m})$ \\
Energy content $^{\mathrm{a}}$ & 0.76 & 0.97 & $\mathrm{~J}$ \\
Power dissipation $^{\mathrm{a}, \mathrm{b}}$ & 0.72 & 0.88 & $\mathrm{~W}$ \\
\hline \hline
\end{tabular}

${ }^{\mathrm{a} A t} E_{\mathrm{acc}}=1 \mathrm{MV} / \mathrm{m}$ and reference length $(3 / 2) \beta_{0} \lambda$.

${ }^{\mathrm{b}} R_{s}=73 \mathrm{n} \Omega$.

TABLE VI. Radio-frequency properties, $325 \mathrm{MHz}, \beta_{0}=0.82$ and $\beta_{0}=1$ cavities optimized for higher shunt impedance.

\begin{tabular}{lccc}
\hline \hline Parameter & $\beta_{0}=0.82$ & $\beta_{0}=1$ & Units \\
\hline Energy gain at $\beta_{0}{ }^{\mathrm{a}}$ & 1135 & 1384 & $\mathrm{kV}$ \\
$R / Q$ & 640 & 743 & $\Omega$ \\
$Q R_{s}$ & 171 & 183 & $\Omega$ \\
$(R / Q) \cdot Q R_{s}$ & $1.09 \times 10^{5}$ & $1.36 \times 10^{5}$ & $\Omega^{2}$ \\
$E_{p} / E_{\text {acc }}$ & 4.13 & 4.11 & $\cdots$ \\
$B_{p} / E_{\text {acc }}$ & 7.54 & 7.77 & $\mathrm{mT} /(\mathrm{MV} / \mathrm{m})$ \\
$B_{p} / E_{p}$ & 1.83 & 1.89 & $\mathrm{mT} /(\mathrm{MV} / \mathrm{m})$ \\
Energy content $^{\mathrm{a}}$ & 1.01 & 1.25 & $\mathrm{~J}$ \\
Power dissipation $^{\mathrm{a}, \mathrm{b}}$ & 0.80 & 0.96 & $\mathrm{~W}$ \\
\hline \hline
\end{tabular}

${ }^{\mathrm{a} A t} E_{\text {acc }}=1 \mathrm{MV} / \mathrm{m}$ and reference length $(3 / 2) \beta_{0} \lambda$.

${ }^{\mathrm{b}} R_{s}=68 \mathrm{n} \Omega$.

cannot argue that this is universally true, only that there appears to be a correlation present.

From the previous sections, there are some generalizations that can be made with respect to maximizing the shunt impedance. (i) Increasing the spoke base length and decreasing the width increases the shunt impedance (Figs. 5 and 9), but can also increase the peak magnetic field. (ii) The shunt impedance can be increased by making the spoke aperture length and width close to equal. (ii) Increasing the parameter $h \_o u t$ helps to increase the shunt impedance (Fig. 27)

In Tables V and VI, we present the rf properties for all four cavities given in the previous section; however, here the focus of optimization was to achieve a higher shunt impedance while still maintaining reasonable surface fields.

\section{CONCLUSION}

High-velocity spoke cavities can be optimized to provide low power dissipation (high shunt impedance) and surface fields which allow for reasonable accelerating gradients. The minimization of the surface fields by increasing the dimensions of the spoke base causes the cavity to lose some of its compactness, but still maintains transverse dimensions of around $20 \%$ less than that of a TM cavity at the same frequency. Depending on the application (e.g. if only low gradients are required), a cylindrical or longitudinally oriented spoke can be used to regain the full compactness advantage.

High-velocity spoke cavities have a more complicated geometry than their TM counterparts, and as such, have a large number of variable parameters that allow for customization for a variety of applications. This makes it difficult to determine a few simple rules for optimization, but here we have identified the most influential parameters and how they can be used to reduce the peak surface fields and increase the shunt impedance. If the fields are to maintain a proper balance, for the geometries studied here, it was found that the trade-off between normalized fields and shunt impedance is at least $10 \%$. In other words, the shunt impedance of a cavity can be increased by at least $10 \%$ at the expense of increasing the normalized electric and magnetic fields by $10 \%$.

The optimized multispoke geometries presented here lead to all accelerating gaps being of similar length because the transversely oriented spoke base is large enough to decrease the cell-to-cell coupling. If we define this coupling as in [44],

$$
k=\frac{\frac{1}{2}\left[\left(f^{(N)}\right)^{2}-\left(f^{(1)}\right)^{2}\right]}{2\left(f^{1}\right)^{2}-\left(f^{N}\right)^{2}\{1-\cos [\pi / N]\}},
$$

where $f^{1}$ is the frequency of the fundamental mode and $f^{N}$ is the frequency of the nearest mode $(N=2)$. Figure 38 is an example of how the cell-to-cell coupling $k$ decreases as the transverse dimension of the spoke base increase, and stays relatively the same as the base dimensions of a longitudinally oriented spoke increase. This is an important point that should be emphasized again. A longitudinal spoke base orientation allows for a much larger mode separation and slightly smaller diameter, which is why,

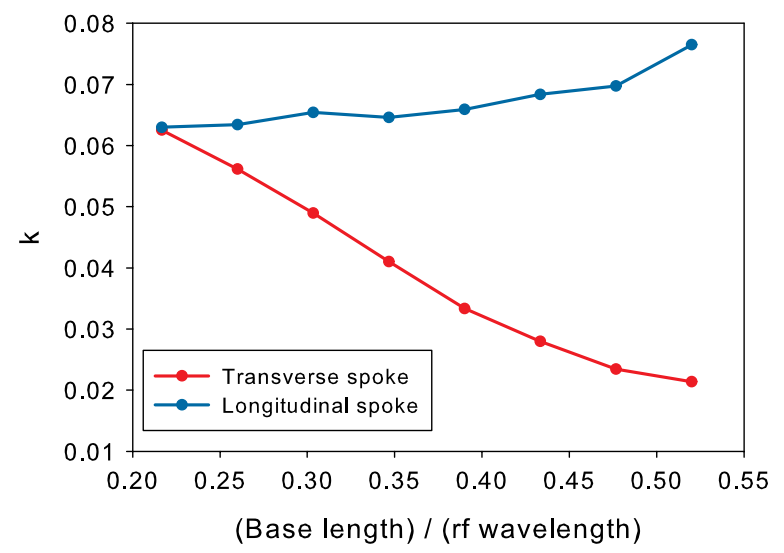

FIG. 38. Coupling $k$ between cells of an optimized $\beta_{0}=0.82$, $325 \mathrm{MHz}$ two-spoke cavity. The leftmost point is common to both curves since it represents a cylindrical spoke base. 


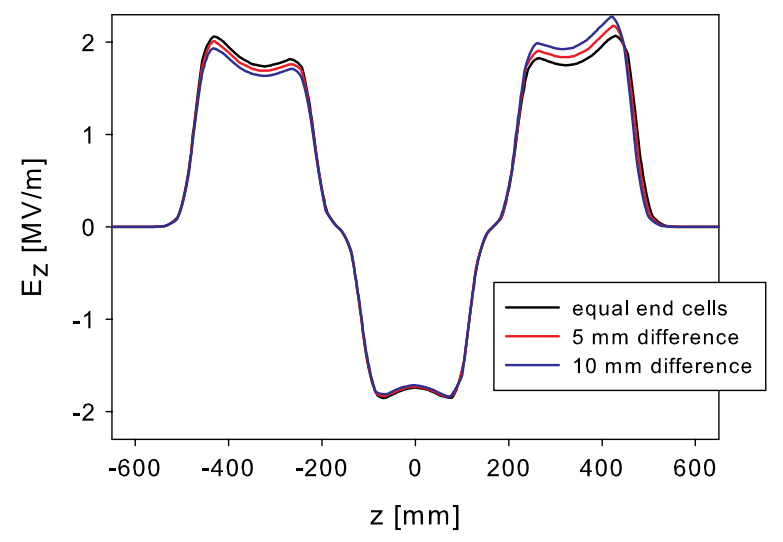

FIG. 39. On-axis electric field profile for equal end cell lengths and an imbalance of 5 and $10 \mathrm{~mm}$, for an optimized $325 \mathrm{MHz}$, double-spoke cavity. The imbalances are created by adding length to one end cell while keeping the spokes fixed.

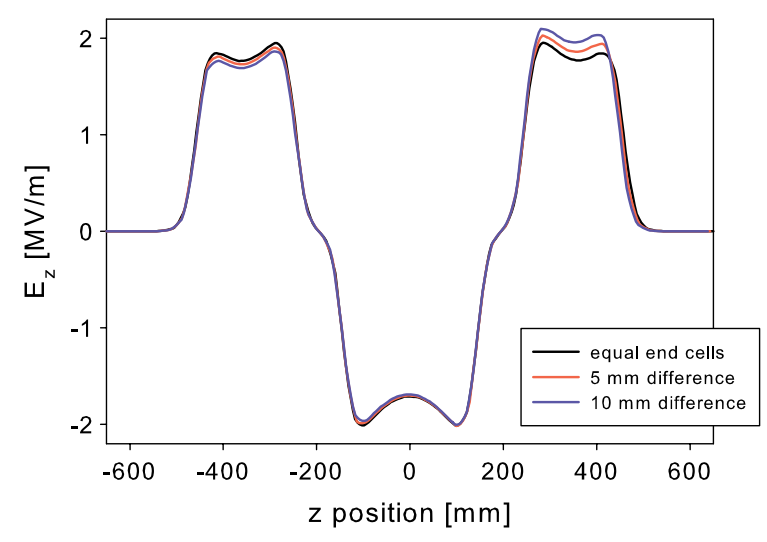

FIG. 40. On-axis electric field profile for equal end cell lengths and an imbalance of 5 and $10 \mathrm{~mm}$ for a double-spoke cavity with cylindrical spokes, operating at $325 \mathrm{MHz}$. The imbalances are created by adding length to one end cell while keeping the spokes fixed.

for some applications, they may be preferable to the transverse geometries presented here.

This decreased cell-to-cell coupling accompanying an optimized spoke design would suggest that perhaps tuning to achieve field profile balance could become important, as it is in TM cavities. However, even with only a few percent coupling, the tolerance to manufacturing inaccuracies is quite high. Figure 39 shows that with a $3 \%$ coupling, a $5 \mathrm{~mm}$ imbalance in the length of the end cells leads to a $10 \%$ difference in the on-axis electric field while for as much as a $1 \mathrm{~cm}$ imbalance, there is only a $16 \%$ difference in the fields.

By comparison, with a cylindrical spoke geometry, the tolerance to manufacturing inaccuracies is even higher, as would be expected. Figure 40 shows how the on-axis longitudinal electric field changes in each cell when one of the end cells is reduced in length by 5 and $10 \mathrm{~mm}$. As opposed to the field profiles in Fig. 39, where the voltage acquired in each cell is roughly equal, it is clear, as was previously mentioned, the voltage gained in the center cell of the cylindrical spoke cavity (Fig. 40) is roughly twice that of the end cells. The cell-to-cell coupling in this cavity, by Eq. (10), is 6\%. The imbalance of the fields is now $3.8 \%$ and $7.3 \%$ for a cell length difference of 5 and $10 \mathrm{~mm}$, respectively.

What can be concluded from this analysis is that spoke cavities are highly customizable. Optimizing them for low peak surface fields and high shunt impedance does lessen some of their advantages outlined previously-their transverse size increases and the separation between the fundamental and nearest modes decreases-however those advantages are still present, and can be quite significant depending on the application.

\section{ACKNOWLEDGMENTS}

We would like to thank Subashini De Silva, HyeKyoung Park, and Rocio Olave for their assistance with the various simulation software used in this research and useful discussions. This work has been supported by the U.S. Department of Energy Award No. DE-SC0004094. This research used resources of the National Energy Research Scientific Center, which is supported by the Office of Science of the U.S. Department of Energy under Contract No. DE-AC02-05CH11231.

[1] M. Kuntze, in Proceedings of the 1973 Particle Accelerator Conference, San Francisco, CA [IEEE Trans. Nucl. Sci. 20, No. 3 (1973)].

[2] I. Ben-Zvi and J. M. Brennan, Nucl. Instrum. Methods 212, 73 (1983).

[3] J. R. Delayen, Nucl. Instrum. Methods Phys. Res., Sect. B 40/41, 892 (1989).

[4] J. R. Delayen, C. L. Bohn, and C. T. Roche, Nucl. Instrum. Methods Phys. Res., Sect. B 56/57, 1025 (1991).

[5] J. R. Delayen and C. L. Bohn, in Proceedings of the 4th Workshop on RF Superconductivity (KEK, Ibaraki, 1990), p. 427 [http://accelconf.web.cern.ch/accelconf/SRF89/ papers/srf89g01.pdf].

[6] J.R. Delayen, W.L. Kennedy, and C.T. Roche, in Proceedings of the 1992 Linear Accelerator Conference (Love Printing Services, Ontario, Canada, 1992), p. 695 [http://accelconf.web.cern.ch/accelconf/192/papers/th4-32 .pdf].

[7] K. W. Shepard, P. N. Ostroumov, and J. R. Delayen, Phys. Rev. ST Accel. Beams 6, 080101 (2003).

[8] J.R. Delayen, in Proceedings of the 10th Workshop onRF Superconductivity, edited by S. Noguchi (KEK, Tsukuba, Japan, 2001), pp. 152-161 [http:// accelconf.web.cern.ch/accelconf/srf01/papers/fa007.pdf].

[9] M. Kelly, in Proceedings of the 13th International Workshop on RF Superconductivity, edited by J. K. Hao, S. L. Huang, and K. Zhao (PKU, Beijing, China, 2007), p. WE302 [http://accelconf.web.cern.ch/accelconf/srf2007/ PAPERS/WE302.pdf]. 
[10] S. Belomestnykh and V. Shemelin, in Proceedings of the 12th International Workshop on RF Superconductivity, edited by H. Padamsee (Cornell University, Ithaca, NY, 2005), pp. 060424-03 [http://accelconf.web.cern.ch/ accelconf/SRF2005/papers/sua02.pdf].

[11] Z. Conway, K. W. Shepard, M. P. Kelly, J. D. Fuerst, and M. Kedzie, in Proceedings of the 12th International Workshop on RF Superconductivity (Ref. [10]) [http:// www.lns.cornell.edu/public/SRF2005/pdfs/TuA06.pdf].

[12] Z. Conway, M. P. Kelly, S. I. Sharamentov, K. W. Shepard, G. K. Davis, J. R. Delayen, and L. R. Doolittle, in Proceedings of the 13th International Workshop on RF Superconductivity (Ref. [9]), p. WEP67 [http://accelconf.web.cern.ch/ accelconf/srf2007/PAPERS/WEP67.pdf].

[13] M. P. Kelly, K. W. Shepard, and M. Kedzie, in Proceedings of the 10th Workshop on RF Superconductivity (Ref. [8]), p. PT017 [http://accelconf.web.cern.ch/accelconf/srf01/ papers/pt017.pdf].

[14] M. Kelly, in Proceedings of the 2006 Linear Accelerator Conference, edited by S. Henderson (ORNL, Oak Ridge, TN, 2006), MO3002, p. 834 [http://accelconf.web.cern .ch/accelconf/106/PAPERS/MO3002.PDF].

[15] J. R. Delayen, in Proceedings of the 2010 Linear Accelerator Conference, edited by M. Ikegami (KEK, Tsukuba, Japan, 2010) [http://accelconf.web.cern.ch/ accelconf/LINAC2010/papers/tu302.pdf].

[16] K. W. Shepard, M. P. Kelly, J. D. Fuerst, M. Kedzie, and Z.A. Conway, in Proceedings of the 2005 Particle Accelerator Conference, Knoxville, TN (IEEE, Piscataway, NJ 2005), p. 4338 [http://accelconf.web.cern.ch/accelconf/ p05/PAPERS/TPPT100.PDF].

[17] J. D. Fuerst, M. Kedzie, M. P. Kelly, K. W. Shepard, and E. Peterson, in Proceedings of the 2003 Particle Accelerator Conference, Portland, Oregon (IEEE, New York, 2003), p. TPAB044, http://accelconf.web.cern.ch/accelconf/p03/ PAPERS/TPAB044.PDF.

[18] T. Tajima et al., in Proceedings of the 2003 Particle Accelerator Conference, Portland, Oregon (Ref. [17]), p. TPAB063 [http://accelconf.web.cern.ch/accelconf/p03/ PAPERS/TPAB063.PDF].

[19] G. Olry et al., in Proceedings of the 12th International Workshop on RF Superconductivity (Ref. [10]), TuP38 [http://accelconf.web.cern.ch/accelconf/SRF2005/papers/ tup38.pdf].

[20] G. Olry et al., in Proceedings of the 11th Workshop on RF Superconductivity, edited by D. Proch (DESY, Hamburg, Germany, 2004), p. TUP55 [http://srf2003 .desy.de/fap/paper/TuP55.pdf].

[21] I. Gonin et al., in Proceedings of the 2008 Linear Accelerator Conference (Victoria, BC, Canada, 2008), p. THP030 [http://accelconf.web.cern.ch/accelconf/ LINAC08/papers/thp030.pdf].

[22] L. Ristori et al., in Proceedings of the 14th International Conference on RF Superconductivity (Berlin, Germany, 2009), p. THPPO011 [http://accelconf.web.cern.ch/ Accelconf/SRF2009/papers/thppo011.pdf].

[23] W. S. Graves, W. Brown, F.X. Kaertner, and D. E. Moncton, Nucl. Instrum. Methods Phys. Res., Sect. A 608, S103 (2009).

[24] T. Satogata, K. Deitrick, J. R. Delayen, B. R. P. Gamage, K. Hernandez-Chahin, C. S. Hopper, G. Krafft, and R. G.
Olave, in Proceedings of the 4th International Particle Accelerator Conference, edited by Zhimin Dai, Christine Petit-Jean-Genaz, Volker RW Schaa, and Chuang Zhang (JACoW, Shanghai, China, 2013), pp. 2292-2294 [http://accelconf.web.cern.ch/accelconf/IPAC2013/papers/ wepwa078.pdf].

[25] T. Hayakawa, N. Kikuzawa, R. Hajima, T. Shizuma, N. Nishimori, M. Fujiwara, and M. Seya, Nucl. Instrum. Methods Phys. Res., Sect. A 621, 695 (2010).

[26] M. Sawamura, R. Hajima, R. Nagai, and N. Nishimori, in Proceedings of the 15th International Conference on $R F$ Superconductivity, edited by M. Power (JACoW, Chicago, IL, 2012), p. MOPO036 [http://accelconf.web.cern.ch/ accelconf/SRF2011/papers/mopo036.pdf].

[27] P. Ostroumov, at the 4th Meeting of the ESSS Linac Reference Group (2009).

[28] J.R. Delayen, S. U. DeSilva, and C.S. Hopper, in Proceedings of the 2011 Particle Accelerator Conference, New York, NY (IEEE, New York, 2011), p. TUP100 [http://accelconf.web.cern.ch/accelconf/ PAC2011/papers/tup100.pdf].

[29] J. R. Delayen, in Proceedings of the 11th Workshop on RF Superconductivity (Ref. [20]) [http://srf2003.desy.de/fap/ paper/TuT01.pdf].

[30] E. Zaplatin, in Proceedings of the 12th International Workshop on RF Superconductivity (Ref. [10]), TUP41 [http://accelconf.web.cern.ch/accelconf/SRF2005/papers/ tup41.pdf].

[31] F. L. Krawczyk, K. C. D. Chan, R.C. Gentzlinger, W. B. Haynes, J. P. Kelley, D. I. Montoya, E. N. Schmierer, D. L. Schrange, and T. Tajima, in Proceedings of the 8th European Particle Accelerator Conference, Paris, France (EPS-IGA and CERN, Geneva, 2002), pp. 272274 [http://accelconf.web.cern.ch/accelconf/e02/PAPERS/ WEBLA003.pdf].

[32] K. W. Shepard, Z. A. Conway, J. D. Fuerst, M.P. Kelly, G. J. Waldschmidt, and A.M. Porcellato, in Proceedings of the 2007 Particle Accelerator Conference, Abuquerque, New Mexico (IEEE, New York, 2007), p. WEPMN087 [http://accelconf.web.cern.ch/ accelconf/p07/PAPERS/WEPMN087.PDF].

[33] E. Rampnoux, L. Berthelot, P. Blanche, S. Bousson, J. Lesrel, L. Lukovac, and G. Olry, in Proceedings of the 2009 Particle Accelerator Conference, Vancouver, BC, Canada (IEEE, Piscataway, NJ 2009), p. WE5PFP029 [http://accelconf.web.cern.ch/accelconf/pac2009/papers/ we5pfp029.pdf].

[34] Xiao Yong-Chaun et al., Chinese Phys. C 35, 188 (2011).

[35] CST, http://www.cst.com/Content/Products/MWS/Overview .aspx.

[36] J. R. Delayen and L.H. Harwood, in Proceedings of the 2003 Particle Accelerator Conference, Portland, Oregon (Ref. [17]), p. MPPB081 [http://accelconf.web.cern.ch/ accelconf/p03/PAPERS/MPPB081.PDF].

[37] B. Mustapha, P. Ostroumov, and Z. Conway, in Proceedings of the 3rd International Particle Accelerator Conference, New Orleans, LA (IEEE, Piscataway, NJ 2012), p. WEPPC037 [http://accelconf.web.cern.ch/ accelconf/IPAC2012/papers/weppc037.pdf]. 
[38] C.S. Hopper, R. G. Olave, and J. R. Delayen, in Proceedings of the 3rd International Particle Accelerator Conference, New Orleans, LA (Ref. [37]), p. WEPPC103 [http://accelconf.web.cern.ch/accelconf/IPAC2012/papers/ weppc103.pdf].

[39] R. G. Olave, J. R. Delayen, and C.S. Hopper, in Proceedings of the 2012 Linear Accelerator Conference (JACoW, Tel Aviv, Israel, 2012), p. MOPB072 [http://accelconf.web.cern.ch/accelconf/ LINAC2012/papers/mopb072.pdf].

[40] H. Padamsee and A. Joshi, J. Appl. Phys. 50, 1112 (1979).
[41] K. Ko et al., in Proceedings of the 2010 Linear Accelerator Conference (Ref. [15]), p. FR101 [http://accelconf.web .cern.ch/accelconf/LINAC2010/papers/fr101.pdf].

[42] C.S. Hopper and J.R. Delayen, in Proceedings of the 2012 Linear Accelerator Conference (Ref. [39]), p. MOPB056 [http://accelconf.web.cern.ch/accelconf/ LINAC2012/papers/mopb056.pdf].

[43] T. P. Wangler, RF Linear Accelerators (John Wiley and Sons, Inc., New York, 2008), p. 44.

[44] H. Padamsee, J. Knobloch, and T. Hays, RF Superconductivity for Accelerators (John Wiley and Sons, Inc., New York, 1998). 